\title{
I. Auf der Suche nach der „evolutionären Lösung": Arbeit an der Fürsorgerechtsreform bis zum zweiten Referentenentwurf vom März 1959
}

Während sich seit Anfang 1956 die sozialpolitische Aufmerksamkeit zunehmend auf die Rentenreform richtete, wurde in der Sozialabteilung des Bundesinnenministeriums in aller Stille bereits an ersten Entwürfen für ein Bundesfürsorgegesetz gearbeitet. Für den neuen Abteilungsleiter Scheffler ergaben sich aus den bisherigen Fachdiskussionen, der Reduktion der „Sozialreform“ auf die Rentenversicherung, aus der zunehmenden wirtschaftlichen Prosperität und tendenziell sinkenden Bedeutung fürsorgerischer Leistungen für den Lebensunterhalt, schließlich aus den bisher schon unternommenen fürsorgerechtlichen Modernisierungsschritten als Reformziele:

- eine Zusammenfassung und Systematisierung des Fürsorgerechts;

- eine Intensivierung fürsorgerischer Tätigkeit durch Schwerpunktverlagerung auf die individuelle, über die bloße Existenzsicherung hinausgehende personale Hilfestellung für breitere Bevölkerungskreise;

- eine stärkere Verrechtlichung der Fürsorge, wobei jedoch die klassischen Leitprinzipien des Nachrangs und der Individualisierung gewahrt werden sollten;

- eine bessere Sicherung der finanziellen und personellen Leistungsfähigkeit der Träger der öffentlichen Fürsorge;

- eine sinnvolle Verzabnung der Leistungen der Fürsorge mit denjenigen anderer Sicherungsträger;

- in Konsequenz aus alledem eine deutliche Verbesserung des Images der öffentlichen Fürsorge. ${ }^{1}$

Mit Vollbeschäftigung und Ausbau der Sozialversicherung, so Achinger auf dem Fürsorgetag 1957, bestünde nun die Chance „für eine Wiederherstellung und Ausbildung der hohen Ziele, die nach dem ersten Weltkrieg postuliert, aber nur zum kleinen Teil verwirklicht wurden". ${ }^{2}$ Diese Ziele waren unter denjenigen, die eine Reform des Fürsorgerechts befürworteten, wenig kontrovers. Ebensowenig gab es wirksame Widerstände gegen eine Reform des Fürsorgerechts schlechthin. ${ }^{3}$ Umso mehr kann es verwundern, daß von den ersten Vorarbeiten bis zur Vorlage überhaupt eines offiziellen ersten Referentenentwurfs gut zweieinhalb Jahre, bis zur Kabinettsreife dann weitere anderthalb Jahre vergingen, dies ohne Regierungs- oder Ministerwechsel und bei stabilen parlamentarischen Mehrheiten.

Eine der wesentlichen Ursachen dafür war, daß die herkömmliche Fürsorge aufgrund ihrer traditionell depravierten bzw. diffusen Klientel - wer rechnete da-

Vgl. Anlage zu Scheffler an Heusler etc. am 9.2.1956, BAK, B 106/9789/2.

Achinger, Neuordnung des Fürsorgerechts, S. 41.

3 Vgl. auch Buhr u.a., Armutspolitik, S. 509ff., 529. 
mit, künftig einmal Fürsorge zu benötigen? - verglichen etwa mit der Sozialversicherung oder der Kriegsopferversorgung kaum attraktive politische Profilierungsmöglichkeiten bot, zumal für einen Ressortleiter, der ohnehin eher außenpolitische Ambitionen hegte. Dementsprechend fehlte, anders als bei der Rentenreform und im Bundesinnenministerium zunächst auch für die Fürsorge befürchtet, massiver Druck von seiten der sozialdemokratischen Opposition, und die dringend reformbedürftige Körperbehinderten- und Tuberkulosefürsorge wurden vorerst gesondert neu geregelt. So blieb die Fürsorgereform die meiste Zeit ein Thema für Spezialisten, das politisch wie regierungsintern lange nur auf unterer und mittlerer Ebene verhandelt wurde, ehe mit dem Subsidiaritätsstreit eine sachlich eigentlich zweitrangige Frage seit 1959 auch die politische Führungsspitze für das Gesetz interessierte.

Zwar forderte der Bundestag im Januar 1957 schließlich die baldige Vorlage eines Entwurfs für ein neues Fürsorgegesetz ${ }^{4}$; doch bis zur Bundestagswahl 1957 dominierte ohnehin die Rentenreform das öffentliche Interesse und die parteipolitischen Auseinandersetzungen, und auch danach gab es weder von seiten der alten und neuen Bundestagsopposition noch in einer breiteren Öffentlichkeit entsprechende Vorstöße. Im Gegenteil, mit den allseits spürbaren oder zumindest erwarteten Wirkungen des „Wirtschaftswunders“, flankiert durch die individuellen Effekte der Sozialgesetzgebung, mehrten sich in der Union die Stimmen, die wie Eugen Gerstenmaier auf dem CDU-Parteitag im Mai 1957 meinten, „auf der äußersten Grenze“ zu stehen, „die den Sozialstaat [...] vom haltlosen Gefälligkeitsstaat, ja vom Versorgungsstaat hochsozialistischer Prägung " unterscheide. ${ }^{5}$

Auch das Bestreben des Innenministeriums nach einem möglichst breiten Konsens für das neue Gesetz kostete Zeit. Schließlich bewegte sich der Bundesgesetzgeber hier im Kräftefeld von Länderzuständigkeit und kommunaler Selbstverwaltung und hing ein Erfolg des Gesetzes entscheidend von der Kooperationsbereitschaft der Bundesländer, der Kommunen und auch der freien Wohlfahrtsverbände ab. Und nicht zuletzt war es die Breite der Materie selbst, für die das fürsorgerische Alphabet von „Altenfürsorge“ bis „Zuständigkeit“ neu buchstabiert werden sollte. Damit bot sich eine Fülle von Ansatzpunkten für grundsätzliche wie detaillierte Kritik und für Konflikte mit der Gesundheitsabteilung im Innenministerium oder dem Familienressort.

Bei der Schilderung der Vorarbeit bis zum Regierungsentwurf vom Februar 1960 zwingt die Breite der Gesetzesmaterie zu darstellerischen Kompromissen: In einem ersten chronologisch angelegten Kapitel soll die Vorgehensweise der Sozialabteilung des Bundesinnenministeriums bis zum zweiten Referentenentwurf vom März 1959 behandelt werden. In den drei weiteren, für diese Arbeit zentralen Kapiteln folgt die Feinanalyse der verschiedenen Reformbereiche, meist über den März 1959 hinaus, um so deren legislatorische Ausgestaltung in den diachronen Zusammenhang einordnen zu können. Ein fünftes Kapitel behandelt dann die erneute Umarbeitung bis zur Verabschiedung des Regierungs-

\footnotetext{
4 Vgl. Sitzung des Bundestages am 21.1.1957; BT, 2. Wp. 1953, Sten. Ber., Bd.34, S. 10599, mit Anlage 15 (Umdruck 912), S. 10617.

5 BldW 105 (1958), S. 5.
} 
entwurfs, die vor allem durch den Konflikt um die Stellung der freien Wohlfahrtspflege geprägt war.

Die Feinanalyse greift dabei immer wieder zurück in die Entstehungszeit des bis 1961 geltenden deutschen Fürsorgerechts. Denn viele Reformziele des BSHG haben tatsächlich ihren Ursprung bereits in den zwanziger Jahren und verweisen damit auf die in jüngerer Zeit auch für den Wohlfahrtsstaatsvergleich entwickelte These der „Pfadabhängigkeit“ spezifischer Formen der Sozialpolitik, die u.a. die „Schwerkraft früherer Sozialpolitik“ durch administrative und institutionelle Beharrungskräfte betont. ${ }^{6}$

Die lange Vorbereitung eines Kabinettsentwurfs war ursprünglich keineswegs geplant. Schon auf dem Fürsorgetag 1955, als Muthesius das ganze Panorama der "Rothenfelser Denkschrift“ entfaltete und ein Gesamtkonzept für eine soziale Neuordnung als Voraussetzung aller Einzelreformen propagierte, hatte Scheffler unter Berufung auf seinen Minister erklärt, daß man mit der Fürsorgereform nicht warten solle, „bis die anderen Bereiche in ihrer Ordnung schon weit fortgeschritten sind". 7 Die wachsende Ungeduld Adenauers mit den dahindümpelnden Sozialreformarbeiten, die auch das Bundesinnenministerium immer wieder dem Arbeitsressort angekreidet hatte, zeigte also auch im Innenministerium selbst Wirkung: Im April 1956 berichtete die DLT-Geschäftsführung vertraulich von „ernsthaften" Arbeiten dieses Ministeriums an einem Referentenentwurf; man wolle, so hieß es, „im BMI jedenfalls konkrete Schubladenentwürfe haben, falls ,der große Boß` eines Tages ungeduldig werde und auf dem Gebiete des Fürsorgewesens Taten sehen wolle". 8

Die Intensität, mit der in der Sozialabteilung seit dem Winter 1955/56 an der Reform gearbeitet wurde, widersprach allerdings der Verharmlosung als "Schubladenentwürfe". Tatsächlich war unter Schefflers Leitung die Sozialabteilung des Innenministeriums, das wegen der im Dezember 1955 veröffentlichten RichtsatzVorschriften in die öffentliche Kritik geraten war, mittlerweile selbst an einer Systematisierung und Modernisierung des Fürsorgerechts interessiert. ${ }^{9}$ Wichtiges Motiv war auch die Sorge, sonst würden immer mehr Sondergesetze für einzelne Gruppen unvermeidbar, dadurch das Fürsorgerecht immer stärker „aufgesplittert", die Arbeiten für die zuständigen Stellen immer mehr erschwert ${ }^{10}$ und nicht zuletzt die öffentlichen Haushalte immer stärker belastet. Anders als bei den bisherigen Modernisierungsschritten bedurfte es also jetzt nicht mehr äußeren indirekten Drucks, um eine solche Reform zu bewirken. Die Sozialabteilung wurde

\footnotetext{
${ }^{6}$ Dazu vgl. Pierson, Politics, S.14ff.; ferner Conrad, Wohlfahrtsstaaten, S.160; ders., Alterssicherung.

7 Vgl. Fürsorge und Sozialreform, S. 495ff. (Zitat S. 497); Muthesius, Die Fürsorge und die Neuordnung.

8 „Siegburger Monatsbericht“ April 1956 der DLT-Hauptgeschäftsstelle, BAK, B 172/44401/1.

9 Vgl. auch Deutschland im Wiederaufbau, S. 122.

10 Vgl. Bundesinnenminister Schröder bei der ersten Lesung des BSHG-Entwurfs im Bundestag am 4.5.1960, BT, 3. Wp. 1957, Sten. Ber., Bd. 45, S. 6255.
} 
damit zum Motor der Reform, die von einem Teil der Trägerinstitutionen, nämlich vielen kreisfreien Städten, ebenso wie von diesen eng verbundenen Experten wie Muthesius, aber auch den Fachausschüssen des DV gewünscht, von dem anderen Teil der Träger, den Landkreisen, allerdings nach wie vor abgelehnt wurde. Damit eröffnete sich die Chance, über das Interesse der Ministerialbürokratie an der eigenen Imagepflege und am Nachweis der eigenen Kompetenz ebenso wie über das Interesse der städtisch orientierten Fürsorgefachleute an einer Aufwertung bzw. Domänensicherung und rechtlichen Systematisierung der öffentlichen Fürsorge, auch indirekt die Interessen einer tatsächlichen oder potentiellen Fürsorgeklientel zur politischen Durchschlagkraft zu verhelfen. ${ }^{11}$

Bereits Anfang Februar 1956, noch bevor der neue Fürsorgeausschuß des Beirats zum ersten Mal tagte, hatten Scheffler und Gottschick detaillierte Konzepte für die geplanten Individualhilfen und die laufenden Unterhaltshilfen erarbeitet. ${ }^{12}$ Außerdem hatte Scheffler einen Thesen- und Fragenkatalog zusammengestellt, der fast alle wesentlichen Elemente der künftigen Gesetzentwürfe enthielt: die Zusammenfassung des gesamten Fürsorgerechts; eine deutliche Abtrennung der Hilfen für den Lebensunterhalt von den Individualhilfen, die katalogmäßig aufgeführt wurden; für letztere sollte die bisherige enge Definition der Hilfsbedürftigkeit verlassen werden, indem höhere Einkommensgrenzen geprüft wurden, ein Weg, den sein Haus bereits in den Entwürfen für ein Körperbehindertengesetz und ein Gesetz über Tuberkulosehilfe beschritt; die Stärkung der Rechtsposition des Hilfeempfängers; die Wahrung der behördlichen Ermessensräume bei gleichzeitiger Konkretisierung der gesetzlichen Vorgaben; die klare Regelung von Trägerschaft, Zuständigkeit, Kostenausgleich und Verhältnis zur freien Wohlfahrtspflege und anderen Sozialleistungsträgern (möglicherweise in neuen örtlichen Kooperationsmodellen); die Sicherung der Leistungsfähigkeit der Träger, also der kreisfreien Städte und Landkreise; nicht zuletzt die Umbenennung der öffentlichen Fürsorge, etwa in „soziale Hilfe“, um so deren Modernisierung auch nach außen zu demonstrieren. ${ }^{13}$ Dieser Katalog, den Scheffler in der folgenden Zeit verschiedenen Reformgremien vorlegte, diente einerseits der möglichst breiten fachlichen Absicherung und Konsensbildung. Gleichzeitig aber bot er die Möglichkeit, die Arbeitsweise der Gremien zu beeinflussen und den Eindruck zu stützen, die Sozialabteilung sei über das Stadium der Materialsammlung noch nicht hinaus. ${ }^{14}$

11 Die Bedeutung solch „mittelbare[r] Transformationsprozesse“ von Klientelinteressen hält Winter, Interessen, S.439ff., bezogen auf Großverbände wie Gewerkschaften oder Wohlfahrtsverbände für größer als die in der Verbändeforschung vielfach dominierende Perspektive der unmittelbaren Interessentransformation durch Klientenorganisationen (Zitat S. 444).

12 Vgl. das Konzept „C. Sozialhilfe“ vom 29.11.1955, BAK, B 106/20648; die Abteilungsleitervorlagen vom 29.12.1955, BAK, B 106/9689, und 4.2.1956, BAK, B 106/9688; sowie Notizen über die Besprechung mit Abteilungsleiter am 7.2.1956 mit Anlage, ebenda.

13 Vgl. Anlage zu Scheffler an Heusler etc. am 9.2.1956, BAK, B 106/9789/2.

14 Zur wechselseitigen Einflußnahme von Staats- und Kommunalpolitik durch Ausschüsse und Beiräte vgl. nach wie vor Bertram, Staatspolitik, S. $95 f$. 
Als erstes erörterten Scheffler und Gottschick diesen Katalog seit Mitte Februar 1956 allerdings in ausdrücklich informellen und vertraulichen Gesprächen mit drei Männern der Praxis, die über die kommunalen Spitzenverbände vermittelt worden waren, aber nicht als deren offizielle Vertreter fungierten: mit dem Dürener Oberkreisdirektor Eduard Bierhoff, Mitglied im DLT-Sozialausschuß, mit dem Städtischen Direktor Franz Flamm aus Freiburg, Mitglied im Sozialausschuß des DST, und dem Düsseldorfer Beigeordneten Heinz Heusler, der u.a. dem Vorstand des DV angehörte. ${ }^{15}$ Diese frühe und enge Einbindung der Städte und Landkreise und ihrer Spitzenverbände entsprach der bisherigen kommunalen Affinität der Sozialabteilung; überdies war es nur konsequent, wenn die Fürsorge Angelegenheit der kommunalen Selbstverwaltung bleiben und damit die Umsetzung des Gesetzes entscheidend von den kommunalen Trägern abhängen würde, diese in das Boot der Fürsorgereform zu holen. So nahmen Scheffler und Gottschick meist an den Sitzungen der Sozialausschüsse des DST und des DLT teil, pflegten gerade zu verschiedenen Mitgliedern des DST-Sozialausschusses enge fachliche Kontakte ${ }^{16}$, und Gottschick versorgte den Beigeordneten für Soziales des DLT, Johann Bangert, immer wieder mit wohldosierten vertraulichen Informationen über den Gang der Reformarbeiten. ${ }^{17}$ Außerdem sicherte er zu, daß DST und DLT rechtzeitig über einen Gesetzentwurf informiert würden. ${ }^{18}$

Doch diese Kooperation nur als Einfallstor für Kommunalinteressen zu interpretieren, griffe zu kurz. Zunächst benötigte die Sozialabteilung den kommunalen Sachverstand, um praktische Auswirkungen der einzelnen Reformpunkte eruieren und - nicht weniger wichtig - möglichst rasch konkrete Regelungen finden zu können. ${ }^{19}$ Ferner nutzten Scheffler und Gottschick diese Kontakte ihrerseits immer wieder, um die kommunalen Spitzenverbände auf die eigene Linie zu bringen. Und schließlich war, wie noch zu zeigen ist, gerade Scheffler nicht gewillt, retardierenden Wünschen von kommunaler Seite einfach nachzugeben. In den insgesamt vier Gesprächen bis Anfang Oktober 1956 jedenfalls stießen die Pläne Schefflers und Gottschicks auf weitgehende Zustimmung der kommunalen Fürsorgeexperten; selbst der erfahrene Landkreis-Fachmann Bierhoff versuchte, den geplanten großzügigen Ausbau der einzelnen Individualhilfen allenfalls zu bremsen,

15 Vgl. Vermerk Bangert vom 18.1.1956, BAK, B 172/444-01/4; Vermerk Referat V A 4 vom 24.1.1956, Scheffler an Heusler etc. am 9.2.1956; Vermerk über die Besprechung mit den Sachverständigen am 16.2.1956, BAK, B 106/9789/2.

16 Vgl. die Gespräche mit Marx und Muthesius ab Ende 1956 sowie Scheffler an Schräder am 15.6.1956, BAK, B 106/20652.

17 Vgl. die Vermerke Bangerts über Gespräche mit Gottschick vom 13.4. und 25. 5.1956, BAK, B 172/444-01/4; Bangert an Hans von Koch am 19.5.1956, BAK, B 172/444-01/2. Scheffler kannte den ehemaligen SS-Obersturmbannführer und Reichsamtsleiter in der Kanzlei der NSDAP Bangert aus ihrer gemeinsamen Zeit in der Kommunalabteilung des Reichsinnenministeriums; vgl. Heisig, Armenpolitik, 1990, S.526ff. Inwieweit er über Gottschicks Kontakte zu Bangert informiert war, ist unklar.

18 Vgl. Vermerk Bangert vom 25.5. 1956, BAK, B 172/444-01/1.

19 Daß von seiten des DV-Vorsitzenden solche Unterstützung vorerst nicht zu erwarten war, hatte dieser im Januar 1956 deutlich gemacht, als er auf die Bitte des BMI, mögliche Namen für solche Gespräche mit Praktikern zu nennen, nicht reagierte; vgl. Vermerk Referat V A 4 vom 18.1.1956, BAK, B 106/9789/2. 
plädierte aber etwa bei der im DLT heiklen Frage der Unterhaltspflicht selbst für eine Milderung. Einfluß auf die konkrete Entwurfsgestaltung hatten die Sachverständigen ohnehin stärker auf formalrechtlichem Gebiet, indem sie z.B. erfolgreich gegen die Einführung eines unabhängigen Entscheidungsgremiums im Fürsorgeamt oder gegen die Aufgabendelegation an freie Wohlfahrtsverbände votierten. ${ }^{20}$

Am 21. Februar 1956 tagte unter Vorsitz von Muthesius erstmals auch der neue "Arbeitsausschuß für Fragen der Fürsorge“ des Beirats für die Neuordnung der sozialen Leistungen. Seine vom Innenministerium nominierten Mitglieder waren allesamt ausgewiesene Kenner der Materie, mehrheitlich Mitglied in Hauptausschuß oder Vorstand des DV und repräsentierten die breite Palette der mit der Durchführung der künftigen Fürsorge befaßten Stellen: die Regierungsdirektorin Käthe Petersen von der Hamburger Sozialbehörde als Vertreterin der Länder, bis zu seinem plötzlichen Tod im August 1956 der stellvertretende Ausschußvorsitzende Kitz, der Frankfurter Sozialdezernent Prestel (CDU), der eng mit Muthesius kooperierende und bis zu seinem Unfalltod im Juli 1958 wohl in seinem Verband aktivste Fürsorgespezialist Theodor Marx ${ }^{21}$ für den DST, der Flensburger Landrat Hartwig Schlegelberger (CDU) für den DLT, der Leiter des niedersächsischen Landessozialamts Wyneken Kobus für die Arbeitsgemeinschaft der Landesfürsorgeverbände, Paul Collmer und die Vorsitzende des Katholischen Fürsorgevereins für Mädchen, Frauen und Kinder (KFV) und stellvertretende Vorsitzende der Frauenvereinigung in der CDU, Elisabeth Zillken ${ }^{22}$ für die freie Wohlfahrtspflege, der Fürsorgebeauftragte des DGB Walter Henkelmann sowie als Vertreter von Nachbarbereichen der Leiter des Bielefelder Kreisgesundheitsamtes Alfred Rainer, der Direktor der Landesversicherungsanstalt Oberbayern Lang und Valentin Siebrecht, Abteilungsleiter in der Nürnberger Bundesanstalt für Arbeit. 23

An den insgesamt zwanzig, meist zweitägigen Sitzungen des Ausschusses bis zum Sommer 1958 nahmen neben Scheffler bzw. seinem Nachfolger Duntze und Gottschick in der Regel auch zahlreiche Vertreter der Bundesministerien für Arbeit, für Finanzen und für Vertriebene sowie auch einzelne Mitglieder des Beirats, vor allem Auerbach, teil. Doch nicht nur die Größe des vertraulich tagenden Ausschusses garantierte bereits eine gewisse Behäbigkeit der Arbeitsweise. Auch der Vorsitzende Muthesius selbst drosselte das Beratungstempo: Anders als Scheffler, der bei der ersten Sitzung den Inhalt seines Thesenpapiers zur Diskussion stellte und die „Erarbeitung konkreter Grundsätze für die gesetzgeberische Tätigkeit“

20 Vgl. die Vermerke über die Besprechungen mit den Sachverständigen am 16.2., 14.3., 3.5. und 9.10.1956, Scheffler an Bierhoff etc. am 11.4. und 22.9.1956, mit Anlagen, ebenda.

${ }^{21}$ Marx, bis Ende Mai 1956 in Nürnberg Stadtrat für Sozialfürsorge und Jugendbelange, gehörte neben dem Sozialausschuß des DST auch dem Vorstand und diversen Fachausschüssen des DV an, stellte dort für den DST wichtige Querverbindungen her und leistete wie im Beirats-Fürsorgeausschuß wichtige konzeptionelle Vorarbeiten.

$22 \mathrm{Zu}$ Zillken vgl. auch Mockenhaupt, Elisabeth Zillken.

23 Vgl. Niederschrift über die Sitzung des Arbeitsausschusses für Fragen der Fürsorge am 21.2.1956, ADW, HGSt 6769. 
für vordringlich hielt, gewann Muthesius den Ausschuß dafür, zunächst einmal „den Gesamtkomplex laufender Leistungen“ zu beraten. ${ }^{24}$ Damit hatten sich die alten Fronten verkehrt: Während die Bundesregierung die umfassende Sozialreform bereits „auf ein bald zum Stillstand führendes Nebengleis" 25 geschoben hatte und nun das Innenministerium die lange von ihm blockierte Fürsorgereform forcierte, forderte mittlerweile der DV-Vorsitzende und Rothenfelser Gutachter nachdrücklich einen konzeptionellen Rahmen, „der die Einheitlichkeit der gesamten Neuordnung verbürgt" und am besten vom Bundeskanzler in einer Proklamation verkündet werden sollte. ${ }^{26}$ Anders als Anfang der fünfziger Jahre fürchtete Muthesius nicht mehr, die Fürsorge werde von der "Sozialreform“ mehr oder weniger ausgenommen, sondern sah in ihr umgekehrt die unabdingbare Voraussetzung für eine Reform der Fürsorge in seinem Sinne. Insofern war auch das Arbeitsprogramm des Fürsorgeausschusses nur konsequent. ${ }^{27}$ Dem Bemühen um eine Gesamtkonzeption entsprach es auch, daß der Ausschuß bis zum Herbst 1956 um eine Abstimmung mit den Ergebnissen der übrigen Beirats-Arbeit bemüht war und sich mit übergreifenden Grundsatzfragen wie der Rehabilitation befaßte. ${ }^{28}$ Allerdings lief der Ausschuß damit Gefahr, statt die entscheidende Vorarbeit für die Fürsorgereform zu leisten, hinter den Arbeiten im Innenministerium hinterher zu hinken. Dort jedenfalls, kolportierte Bangert im April 1956 Äußerungen Gottschicks, laufe die Arbeit am Referentenentwurf „völlig selbständig“ neben den Beratungen des Fürsorgeausschusses, der „augenscheinlich sehr langatmig arbeiten werde“.29

Auch Scheffler war sich bewußt, daß er für einen sorgfältig ausgearbeiteten Gesetzentwurf Zeit benötigte, und war daher erleichtert, daß der CDU-Parteitag Ende April keine entsprechenden Forderungen erhob. ${ }^{30}$ Vielmehr wurde dort die Fürsorge vergleichsweise knapp und konzeptionslos abgehandelt. ${ }^{31}$ Wenn Scheffler die Vorarbeiten trotzdem weiter vorantrieb, dann, um nicht wie das Arbeitsministerium durch Initiative des Kanzlers oder jüngst durch einen eigenen Entwurf der SPD überrumpelt zu werden. ${ }^{32}$ Ein weiteres, in den Unterlagen aller-

${ }^{24}$ Ebenda; ferner Niederschrift über die Sitzung des Arbeitsausschusses am 6./7.4.1956, ebenda.

25 Hockerts, Entscheidungen, S. 434.

26 Muthesius, Sozialreform; ähnlich vor der Gruppe II des DV-Studienkreises „Soziale Neuordnung" am 26.1.1956, Niederschrift, ADW, ZB 856.

27 Vgl. Muthesius, Die Fürsorge und die Neuordnung, S. 29.

28 Vgl. die Niederschriften über die Sitzungen des Arbeitsausschusses für Fragen der Fürsorge am 8.5., 15./16.6., 19./20.10. sowie 30.11./1.12.1956, ADW, HGSt 6769.

29 „Siegburger Monatsbericht“ April 1956 der DLT-Hauptgeschäftsstelle, BAK, B 172/44401/1, sowie Vermerk Bangert vom 13.4.1956, BAK, B 172/444-01/4.

30 Vgl. Abteilungsleiter V an Staatssekretär I am 4.5.1956, BAK, B 106/20652.

31 Während für die Reform der Renten- und Krankenversicherung konkrete und kohärente Reformziele benannt wurden, lauteten die Forderungen für die Fürsorge nur: Rechtsanspruch auf Fürsorge, bundesweite Bestimmungen für die laufenden Unterstützungen, Beschränkung von Unterhalts- und Rückerstattungspflicht, Fürsorgelastenausgleich für ärmere Landkreise, Besserstellung des fürsorgerischen Fachpersonals; vgl. Lünendonk, CDU-Parteitag, S. $128 \mathrm{ff}$.

32 Vgl. Vermerke Bangerts über Gespräche mit Gottschick vom 25.5.1956 und 4.2.1957, BAK, B 172/444-01/4. 
dings nicht nachweisbares Motiv war möglicherweise, daß auch in der DDR das Fürsorgerecht reformiert worden war. ${ }^{33}$

Am 25. Mai 1956 war der erste Teilentwurf des „Sozialhilfegesetzes“ fertig. Er befaßte sich mit dem materiellen Recht und statuierte einen Rechtsanspruch auf „Sozialhilfe“. Während sich der Sonderabschnitt über die laufenden Unterstützungsleistungen weitgehend im bisherigen Rahmen bewegte, brachte der dritte Abschnitt zur „Hilfe für besonderen Lebensbedarf“ wesentliche Neuerungen: Mit dem Katalog der Wirtschaftshilfe, Ausbildungshilfe, Krankenhilfe, Hilfe für Schwangere und Wöchnerinnen, Hilfe für körperlich und geistig Behinderte, der Hilfe zur Pflege, der Haushaltshilfe, der Hilfe für sozial Gefährdete, der „Hilfe in sonstigen Fällen“ und der „Beratung in sozialen Fragen“ wurden zwar noch kaum wirklich neue Hilfearten aufgeführt; neu war aber, daß für alle diese Hilfen bis auf die beiden letztgenannten ein Rechtsanspruch vorgesehen, Voraussetzung und Inhalt der Hilfegewährung jeweils detailliert geregelt und mittels deutlich über dem Richtsatz liegender Einkommensgrenzen der potentielle Empfängerkreis erheblich ausgeweitet wurde. ${ }^{34}$

Vier Tage später referierte Scheffler auf der Konferenz der für das Wohlfahrtswesen zuständigen Länderminister (d.h. vor den sie vertretenden leitenden Fürsorgereferenten) in Berlin über eine mögliche Fürsorgereform, „die im wesentlichen unabhängig von der Reform anderer Sozialleistungsträger laufen" werde. ${ }^{35}$ Zwar machte Scheffler deutlich, daß man nicht an den hergebrachten Fürsorgeprinzipien rütteln und die Grenzen der Bundeszuständigkeit achten wolle; da jedoch bisher der behördliche Ermessensraum „in der Praxis häufig nicht annähernd ausgeschöpft" worden sei, sei eine gewisse Konkretisierung erforderlich, die er dann auf der Grundlage des Thesenkatalogs vom Februar durchexerzierte. $\mathrm{Daß}$ dies bereits zum Teil dem Arbeitsstand im Bundesinnenministerium entsprach, verneinte er.

$\mathrm{Ob}$ allerdings, wie Bangert vom DLT annahm, etwa im Fürsorgeausschuß des Beirats tatsächlich nichts von diesen konkreten Arbeiten bekannt war, erscheint fraglich. ${ }^{36}$ Muthesius und Marx gingen ohnehin davon aus, daß in der laufenden Legislaturperiode keine Verabschiedung eines neuen Fürsorgegesetzes durch den überlasteten Bundestag zu erwarten war, zumal für die Fürsorge von einem „umfangreichen und zeitraubenden Reformgeschäft" auszugehen sei. ${ }^{37}$ Statt dessen

33 Mit drei Verordnungen vom 23.2.1956 hatte der Ministerrat der DDR die „Sozialfürsorge“ mit zentral festzulegenden Unterstützungssätzen weiter standardisiert, diese Leistungen deutlich erhöht und die Rückerstattung beseitigt. Gleichzeitig wurden aber die Voraussetzungen für die Fürsorgegewährung zu einem faktischen Arbeitszwang verschärft und damit die weitere Marginalisierung der Fürsorge im sozialen Sicherungssystem der DDR gefördert; vgl. NDV 38 (1958), S. 44; Frerich/Frey, Handbuch, Bd.2, S. 364ff.; Rudloff, Fürsorge, S. $196 \mathrm{ff}$.

34 Vgl. den BSHG-Teilentwurf vom 25. 5.1956, BAK, B 106/20648.

35 Kurzfassung des Referats, BAK, B 106/20652; ferner Kurzprotokoll über die Konferenz am 29.5.1956, BAK, B 106/9789/2.

36 Vgl. Bangert an Schlegelberger am 2.6.1956, BAK, B 172/444-01/4.

37 Marx, Die Städte zur Neuordnung, S. 73; vgl. ferner Marx, Die Städte und die Neuordnung, S.336ff.; Muthesius, Sozialreform, sowie in einem Vortrag vor dem DLT-Sozialausschuß am 5. 9.1956, Ms., BAK, B 106/9697. 
blieb es vorerst auch die Linie der Fürsorgespezialisten im DST, ein umfassendes Neuordnungskonzept einzufordern, bevor eine Reform des Fürsorgerechts darin systematisch einzubauen sei. Denn nur wenn es gelänge, die sozialen Leistungen so systematisch und effektiv zu ordnen, daß die Fürsorge nicht mehr unzureichende Leistungen anderer Bereiche laufend ergänzen müßte, wenn also die Fürsorge für „ihre eigentliche Aufgabe“ frei würde, sei deren Reform überhaupt möglich. ${ }^{38}$ Inwieweit man dabei einen möglichen Regierungswechsel einkalkulierte, blieb allerdings offen.

Auf der DST-Hauptversammlung im Juni 1956 in Essen jedenfalls wurden „Die sozialen Aufgaben der Städte" im Rahmen der Neuordnung nach einem Vortrag von Marx weiträumig abgesteckt. ${ }^{39}$ Dabei hatten der „Sonderbeauftragte für die Neuordnung der sozialen Hilfe" des DST, Kitz, und die DST-Hauptreferentin Schräder weiterhin eine - finanziell abzusichernde - Kompetenzerweiterung der Städte im Sinn. ${ }^{40}$ Marx hingegen ging es um die Fürsorge selbst: Viel zu lange und inadäquat mit der Bewältigung von Massennotständen überlastet, sollte sie nun endlich so eingesetzt werden, daß sie als gleichwertige dritte Säule der sozialen Sicherung genuin nur durch ihre Methoden zu bewältigende Aufgaben vor allem bei der Gesundheitsfürsorge und der Jugendhilfe übernähme, mit den Städten als berufenen Trägern. ${ }^{41}$ Für eine solche Fürsorgereform fehlten aber wesentliche Voraussetzungen: Nicht einmal innerhalb der Fachkreise gelinge es bisher, „von der Vergangenheit der kommunalen Fürsorge so zu abstrahieren, daß Reminiszenzen nicht verhängnisvolle Einflüsse auf das Leitbild einer Fürsorge der $\mathrm{Zu}$ kunft haben würden. Zudem steht die Fürsorge noch allzu sehr unter dem Ausnahmezustand der direkten und indirekten Kriegsfolgen". ${ }^{42}$ Marx spielte also wie Muthesius auf Zeit: Er wollte abwarten, bis Verbesserungen bei der Rentenversicherung, der Arbeitslosenversicherung und -hilfe, wirtschaftlicher Aufschwung und die eingeleitete Reform der Finanzverfassung die kommunalen Träger so spürbar entlasteten, daß diese zu einem großzügigen Ausbau der gesamten Fürsorge bereit und latente Ängste vor einer abermaligen Überlastung durch Massenarbeitslosigkeit überwunden würden.

Aus ganz anderen Motiven arbeitete mittlerweile auch Bangert im DLT an einem Abbau dieser gerade in seinem Verband verbreiteten „Reminiszenzen“. Im JuniHeft der DLT-Zeitschrift forderte er die Landkreisvertreter auf, sich der modernen gesellschaftlichen und sozialpolitischen Entwicklung zu stellen. Der wachsende allgemeine Wohlstand habe auch die Auffassung über die Höhe angemessener Sozialleistungen verändert: „Der Anspruch an das Leben ist heute weder von Kalorien noch von der unentbehrlich-primitiven Wohnungseinrichtung her abzustecken. Das Existenzminimum ist gemeinsam mit der allgemein gestiegenen Lebenshaltung

38 Vgl. Marx, Die Städte zur Neuordnung, S.21ff. (Zitat S.24); auch DST-Präsident Otto Suhr, Städte, S. 339.

39 Vgl. Der Städtetag 9 (1956), S. $327 \mathrm{ff}$.

40 Vgl. Vortrag Kitz vor dem DLT-Sozialausschuß am 12.1.1956, Ms., BAK, B 172/44401/1; Schräder, Weg; dies., Neuordnung.

41 Vgl. das der Städteversammlung vorgelegte kommunale Sozialprogramm von Marx, Die Städte zur Neuordnung, hier S. $66 \mathrm{ff}$.

42 Marx, Die Städte und die Neuordnung, S. 337. 
nach oben in Bewegung gekommen [...].“ Wollten die Kommunen ihrem Anspruch auf mehr Eigenständigkeit gerecht werden, könnten sie sich bei der Fürsorge, einem „Kernstück des kommunalen Selbstverwaltungsbereichs“, nicht auf eine pauschale Ablehnung jeglicher Reform beschränken. „Bei aller Anerkennung für Sparsamkeit und Achtung vor dem, was war und organisch geworden ist: der Sozialpolitiker darf nie kurzsichtig, knickerig, spießbürgerlich und eng werden." Sonst liefen die Kommunen Gefahr, im Bundestag kein Gehör zu finden, denn wer „nicht überrascht oder gar überfahren werden will, muß beizeiten gestaltend eingreifen “. ${ }^{43}$ Die Telefonate mit Gottschick zeigten also Wirkung: Sie hatten nicht nur belegt, daß das Innenministerium von einer grundlegenden Reform des Fürsorgerechts nicht abzubringen sein würde, sondern Gottschick hatte auch angeführt, daß im Bundestag die kommunalen Spitzenverbände und selbst die Vertreter seines Hauses als notorische Nein-Sager gälten. ${ }^{44}$ Die von Scheffler geplante Einschränkung behördlicher Ermessensräume verdanke sich vor allem der Praxis in vielen Landkreisen: Während in den Städten meist an Fürsorgefragen interessierte Dezernenten des höheren Dienstes die Sozialämter leiteten, werde von den Landräten die Fürsorge „immer noch als etwas Zweitklassiges gesehen [...], mit dem sich die Inspektoren herumschlagen sollen“. Die konkreteren Regelungen sollten daher „draussen denen, die es anwenden müssen, auch einen kleinen Rückhalt durch das Gesetz geben ". ${ }^{45}$ Inwieweit dies auch eine indirekte Aufforderung Gottschicks an die Landkreise beinhaltete, den Städten nicht das Terrain zu überlassen, bleibe dahingestellt. Seit dem Sommer 1956 befaßte sich jedenfalls auch der DLT-Sozialausschuß mit der Fürsorgereform und machte dabei die Eindämmung des „überall auftauchenden Gesetzesperfektionismus" 46 zu einem seiner Hauptziele.

$\mathrm{Zu}$ einem gemeinsamen Vorgehen der Fürsorgeexperten von DST und DLT kam es trotz mancher etwas halbherziger Versuche nicht. ${ }^{47}$ Zwar hielt Muthesius Anfang September im DLT-Sozialausschuß einen viel beachteten Vortrag über die Neuordnung des Fürsorgerechts, in dem er abermals die Sonderrolle der laufenden Leistungen in den Mittelpunkt stellte und eine Grobgliederung für das kommende Gesetz vorlegte. ${ }^{48}$ Doch die anfängliche Zustimmung im DLT bröckelte bald wieder, und man ging getrennter Wege. Das hing, abgesehen von unterschiedlichen parteipolitischen Präferenzen, vor allem damit zusammen, daß im DST-Sozialausschuß tatsächlich das sozialpolitische Interesse die Finanzierungsfrage dominierte und man hier ohnehin davon ausging, daß sich die Mehrbelastungen durch die Reform für die Städte in vertretbarem Rahmen halten würden. Ganz anders im DLT, wo eine restriktive Fürsorgetradition ebenso wie tatsächliche Strukturschwächen vieler agrarischer Kreise zu berücksichtigen waren

43 Die Selbstverwaltung 10 (1956), S. 136.

44 Vgl. Vermerk Bangert vom 25. 5.1956, BAK, B 172/444-01/4.

45 Ebenda; ferner „Siegburger Monatsberichte“ Mai 1956, BAK, B 172/444-01/1.

46 DV-Geschäftsführer Pense auf der Sitzung des DLT-Sozialausschusses am 13.7.1956, Niederschrift, BAK, B 172/444-01/1.

47 Vgl. etwa Schmerbeck an Kitz am 20.12.1955, ebenda.

48 Vgl. Niederschrift über die Sitzung des DLT-Sozialausschusses am 5./6. September 1956, ebenda, sowie ein Manuskript des Vortrags in: BAK, B 106/9697; auch Muthesius, Bundesfürsorgegesetz. 
und damit an großstädtischen Standards orientierte Normierungen der Fürsorge auf Ablehnung stießen. Um aber den Eindruck bloß fiskalischer Interessen zu verwischen, bemühten die DLT-Vertreter vor allem eine sozialethische Argumentation, in der Pflicht (und Recht) zur Selbsthilfe betont und weite Ermessensräume mit den Erfordernissen der individuell wirksamen Hilfe begründet wurden. ${ }^{49}$

Ende November 1956 war in der Sozialabteilung der erste vollständige „Entwurf eines Gesetzes über die Gewährung von Sozialhilfe" fertig. ${ }^{50}$ Er war von dem Bemühen geprägt, eine umfassende individuelle Hilfestellung der Allgemeinheit auch bei immateriellen Notlagen zu gewährleisten und daher die Rechtsstellung des Hilfeempfängers zu stärken, nicht in jedem Fall den vollen Einsatz der eigenen Mittel zu verlangen, bestimmte neue, teilweise detailliert geregelte Leistungsstandards der „Sozialhilfe“ zu sichern und so die Träger der Fürsorge sehr viel stärker als bisher zu binden. Der Katalog der Individualhilfen wurde um die Hilfe für alte Menschen und die vorbeugende Gesundheitshilfe sowie die traditionelle Hilfe für Minderjährige erweitert. Klare Vorschriften über das einzusetzende Personal und Finanzierungshilfen der Länder sollten die Leistungsfähigkeit der Träger sichern. Damit war die erste Arbeitsphase, in der die wesentlichen Gestaltungselemente des geplanten Regierungsentwurfs festgelegt wurden, abgeschlossen, und die Feinarbeit begann.

Mittlerweile rechneten Scheffler und Gottschick allerdings nicht mehr damit, noch in der laufenden Legislaturperiode den Entwurf zur Kabinettsreife bringen zu müssen. ${ }^{51}$ Am 21. Januar 1957 forderte der Bundestag bei der Verabschiedung der Rentenreform in einem Entschließungsantrag von CDU/CSU und FVP zwar einstimmig die Bundesregierung zur Vorlage eines Entwurfs für ein neues Fürsorgegesetz auf; dies sollte "mit tunlichster Beschleunigung“ geschehen, von einer Fertigstellung noch vor der Bundestagswahl aber war nicht die Rede.52 Doch Gottschick ging davon aus, daß man in der neuen Legislaturperiode nicht mehr lange werde warten können, „da schon von den Parteien die verschiedensten Anzapfungen in dieser Richtung vorlägen" 53 . Scheffler erklärte, das Bundesinnenministerium wolle bis Herbst 1957 einen Referentenentwurf fertigstellen, der wohl im Januar 1958 kabinettsreif sei. ${ }^{54}$

49 Vgl. Johann Bangert, Ersatzpflicht; Die Selbstverwaltung 11 (1957), S. 391.

50 BAK, B 106/20648. Die Bestimmungen der geplanten Gesetze für Körperbehindertenfürsorge und Tuberkulosehilfe sowie für die Kriegsopferfürsorge sollten später eingearbeitet werden. Die jeweiligen Einzelregelungen behandeln die folgenden Abschnitte.

$51 \mathrm{Vgl}$. Vermerk Bangert über ein Gespräch mit Gottschick vom 4.2.1957, BAK, B 172/444-01/4.

52 Vgl. BT, 2. Wp. 1953, Sten. Ber., Bd.34, S. 10599, mit Anlage 15 (Umdruck 912), S. 10617. Leider geben weder die zeitgenössischen Protokolle des Sozialpolitischen Arbeitskreises der CDU/CSU-Fraktion, vgl. ACDP, CDU/CSU-Fraktion AK IV, VIII-005-001/3, noch der Fraktion selbst, vgl. ACDP, Büro des Fraktionsvorstands, VIII-001-1007/3, noch des Sozialpolitischen Ausschusses der Bundespartei, vgl. ACDP, Bundesgeschäftsstelle, VII-004-090/2 und -560/2, Hinweise auf das Zustandekommen dieses EntschlieBungsantrags.

53 Vermerk Bangert vom 4.2.1957, BAK, B 172/444-01/4.

54 Vgl. Entwurf der Niederschrift über die Sitzung des DST-Sozialausschusses am 15./16. 3. 1957, LAB, B Rep. 142-9, 1236. 
Bei ihren weiteren Vorarbeiten lavierte die Sozialabteilung zwischen Skylla und Charybdis: Einerseits war sie den beschriebenen Reformzielen verpflichtet; andererseits galt es, die vielerorts beschränkte kommunale Leistungsfähigkeit und Leistungsbereitschaft nicht überzustrapazieren. Insofern war es wichtig, dem Entwurf möglichst starken Rückhalt durch Fachvertreter zu verschaffen. Dabei agierten Scheffler und Gottschick auf zwei Ebenen: In offiziellen Gesprächen mit den Länderspezialisten und durch ihre regelmäßige Teilnahme an den Sitzungen der Sozialausschüsse von DST bzw. DLT informierten sie sich über die dortige Meinungsbildung, zum Teil zu sehr konkreten Einzelfragen. Scheffler hielt dabei die Fiktion aufrecht, sein Ministerium sei noch in der Phase der Materialsammlung, und legte den Gremien den bekannten, nun an den vorliegenden Gesetzentwurf angepaßten Fragenkatalog vor. ${ }^{55}$ Auf diese Weise würde ungeachtet der Tatsache, daß man in seiner Abteilung schon sehr viel weiter war, der künftige Entwurf als mit den Ländern und den Kommunen abgestimmtes Beratungsergebnis präsentiert werden können. ${ }^{56}$

Daneben gab es eine zweite, inoffizielle Ebene der Expertengespräche: Schon seit Mitte Dezember 1956 trafen sich Gottschick und Scheffler in dessen Wohnung mit Marx und Muthesius zu streng vertraulichen Beratungen über den November-Entwurf. So konnten die Ministerialbeamten ihren Entwurf nicht nur durch zwei ausgewiesene Fürsorgeexperten überprüfen lassen, deren Reformlinie in wichtigen Punkten derjenigen Schefflers entsprach. Gleichzeitig bot sich damit die Möglichkeit, die Reformarbeiten im DV, im Beirat und im DST gewissermaßen an diejenigen des Ministeriums rückzukoppeln. ${ }^{57}$ Für Marx und Muthesius, die ja eigentlich die Arbeit an einem konkreten Entwurf für verfrüht hielten, bot sich umgekehrt die verlockende Chance, in einem noch frühen Stadium auf die Regierungsarbeit Einfluß zu nehmen. Das hinderte sie nicht daran, auch weiterhin in den von ihnen beeinflußten Reformgremien in wichtigen Einzelfragen andere Lösungen als das Bundesinnenministerium zu propagieren. Alles in allem aber befürworteten sie die dort geplanten Regelungen. ${ }^{58}$

Auch die Fürsorgereferenten der Länder stützten mehrheitlich die Linie der Sozialabteilung. ${ }^{59}$ Und selbst der DLT-Sozialausschuß zeigte sich bereit, bis an die Grenze des innerhalb des eigenen Verbandes Vertretbaren zu gehen: Anfang Februar anerkannte er nicht nur, daß sich die Vorstellung von den Aufgaben der

55 Vgl. Schreiben der DLT-Hauptgeschäftsstelle an den Landkreisverband Bayern vom 4. 1. 1957, BAK, B 172/444-01/2; Vermerk Abteilungsleiter V vom 18.1.1957, BAK, B 106/20652; den Fragenkatalog übersandte das BMI u.a. auch an den DST; vgl. Gottschick an Schräder am 14.1.1957, mit Anlage, LAB, B Rep. 142-9, 1282.

56 So auch Scheffler in einem Dankesschreiben an die kommunalen Sachverständigen vom 7.1.1957, BAK, B 106/9789/2.

57 Zur engen Zusammenarbeit zwischen Gottschick und Muthesius beim Dirigieren des Fürsorgeausschusses vgl. auch Gottschick an Muthesius, 13.6.1958, BAK, B 106/9778.

58 Vgl. die Vermerke des Referats V A 4 über die Besprechungen mit Muthesius und Marx vom 21.12.1956, 23.1., 21.2., 16.5.1957, BAK, B 106/20652, sowie vom 12.12.1957, BAK, B 106/20643; Scheffler an Muthesius, 25.4.1957, BAK, B 106/20652.

59 Vgl. die Niederschriften über die Besprechungen mit den Referenten der Länder am 12. 12.1956 und 5./6.2.1957, BAK, B 106/9789/2. 
Fürsorge „im neuen Sozialstaat“ gewandelt hätte und der traditionelle Hilfsbedürftigkeits-Begriff zu erweitern sei, sondern stimmte „im Grundsatz“ auch einem Rechtsanspruch auf Fürsorge zu. ${ }^{60}$ Nur wenige Tage zuvor hatte Gottschick Bangert abermals vor den möglichen Konsequenzen einer obstruktiven Haltung gewarnt: „Wenn jetzt nichts auf dem Wege zu einer Fürsorgeneuordnung getan wird, wird die SPD nach Meinung von Herrn G. eines Tages so neuordnen, dass wir die Fürsorge nicht wiedererkennen" ${ }^{61}$ Die nun eingeleitete Neuordnung sollte nach Auffassung des DLT-Sozialausschusses jedoch so gedrosselt wie möglich vonstatten gehen: Wenn er eine Abschaffung der Kostenersatzpflicht und eine Entschärfung der Unterhaltspflicht ablehnte, die „speziellen Hilfen“ nicht mit Rechtsanspruch versehen, möglichst wenig reglementieren und allenfalls „gewisse Leistungsgruppen" konzedieren wollte, dann zeichneten sich künftige Konfliktlinien deutlich ab. Immerhin hatte der DLT damit schon Anfang Februar 1957 eine Stellungnahme zu Schefflers Katalog fertiggestellt.

Der DST-Sozialausschuß nahm sich mehr Zeit: Nach ersten Überlegungen Mitte Oktober 1956 war man im Januar übereingekommen, die Fürsorgereform „,in nächster Zeit intensiv in Angriff zu nehmen", und bildete einen von Marx geleiteten Arbeitskreis, in dem u.a. Käthe Petersen und bis zum November 1957 auch Muthesius mitarbeiteten. ${ }^{62}$ Dieser Arbeitskreis sollte eine detaillierte Stellungnahme des DST zu den Fragen des Innenministeriums erarbeiten. ${ }^{63}$

Auch der Beiratsausschuß änderte nun seine Strategie: Mit dem Bundestagsbeschluß und Schefflers Ankündigung eines Regierungsentwurfs war die weiträumige theoretische Erörterung von Grundlagenkonzepten obsolet geworden; dies umso mehr, als der Ausschußvorsitzende und sein neuer Stellvertreter Marx über das Tempo des Bundesinnenministeriums bestens informiert waren. So behandelte nun auch der Beirats-Ausschuß Fragen wie die Rechtsstellung des Fürsorgeempfängers oder die Regelung der einzelnen künftigen Individualhilfen. ${ }^{64}$ Das hatte zur Folge, daß Gottschick und Scheffler bei ihrer Überarbeitung des Entwurfs

60 Niederschrift über die Sitzung des DLT-Sozialausschusses am 8.2.1957, BAK, B 172/444-01/1.

61 Vermerk Bangert vom 4.2.1957, BAK, B 172/444-01/4.

62 Ursprünglich hatte Marx diese Vorarbeiten noch abgelehnt; vgl. die Niederschriften über die Sitzungen des DST-Sozialausschusses am 12./13.10.1956, LAB, B Rep. 142-9, 1282, und 11./12.1.1957, LAB, B Rep. 142-9, 1236; Vermerk Abteilungsleiter V vom 18.1. 1957, BAK, B 106/20652.

63 Vgl. Niederschrift über die Sitzung des DST-Sozialausschusses am 15./16.3.1957, LAB, B Rep. 142-9, 1236; Vorschläge von Marx zur Neuordnung des Fürsorgerechts vom 19.2.1957, sowie die Niederschriften über die Sitzungen des DST-Arbeitskreises „Fürsorgerecht" am 7./8.5., 2.9., 16.10. und 7.11.1957, LAB, B Rep. 142-9, 1282. Zu den Reformüberlegungen innerhalb des DST vgl. Heisig, Armenpolitik, 1995, S.139ff.; aufgrund der von ihm verwendeten Quellen konstatiert Heisig im DST entscheidenden „Reformdruck“ und überbewertet damit die Bedeutung dieser Aktivitäten für die Arbeit des BMI; die von ihm behauptete Absicht „des“ DST etwa, „einen eigenen ,Fürsorgerechtsentwurf" zu entwickeln" (S.142), wird durch das von ihm herangezogene Protokoll der Sitzung des DST-Sozialausschusses am 12./13. 10.1956, LAB, B Rep. 142-9, 1282, in keiner Weise belegt.

${ }^{64}$ Vgl. Niederschriften über die Sitzungen des Arbeitsausschusses für Fragen der Fürsorge am 1./2.2., 1./2.3., 3./4.5., 14./15.6. sowie 25./26.10.1957, ADW, HGSt 6769. 
vom November 1956 nicht nur die Einwände von Marx und Muthesius, sondern auch die Überlegungen des Ausschusses stärker als bisher berücksichtigten. ${ }^{65}$

Der DV-Vorstand stellte sich ebenfalls darauf ein, daß die Fürsorgereform „unaufschiebbar" sei, und beschloß im Frühling 1957, den nächsten Fürsorgetag im November ganz diesem Thema zu widmen. ${ }^{66}$ Eine eigens im Mai gebildete, ebenfalls von Muthesius geleitete Untergruppe des Studienkreises „Soziale Neuordnung" bereitete neben den einzelnen DV-Fachausschüssen den Fürsorgetag inhaltlich vor und stellte auch einen Teil der Referenten und Arbeitsgruppenleiter. ${ }^{67}$ Dieses weitere Reformgremium verkörperte nahezu idealtypisch die für die deutsche Fürsorge kennzeichnende enge personelle Verflechtung verschiedener Entscheidungs- und Exekutivebenen: So waren nicht nur mehrere Mitglieder der Gruppe gleichzeitig im Fürsorgeausschuß des Beirats und/oder in den Sozialausschüssen des DST und des DLT tätig, sondern zu ihr gehörten auch Fürsorgereferenten der Länder und wichtige Vertreter der konfessionellen Wohlfahrtspflege sowie als ständige Gäste Scheffler und Gottschick. ${ }^{68}$ Damit trafen sich in unterschiedlich legitimierten und politisch positionierten Reformgremien in unterschiedlicher Konstellation fast immer die gleichen Fürsorgeexperten. Dieser potentielle Einflußgewinn einzelner Fachleute brach sich allerdings an dem jeweils unterschiedlich wirksamen $Z$ wang zum Konsens, so daß etwa die Verfechter einer Besserstellung der freien Wohlfahrtspflege die DV-Gremien nicht für ihre Ziele nutzen konnten.

Ein weiteres Element solcher Verflechtungen gerade im Bereich kommunaler Interessen ist der personelle Austausch zwischen Ministerien und kommunalen Spitzenverbänden. ${ }^{69}$ Jüngstes Beispiel dafür war jetzt Anton Oel, Oberregierungsrat in der Sozialabteilung des Bundesinnenministeriums und langjähriger Mitarbeiter Gottschicks, der Anfang 1957 Beigeordneter für Soziales im DST wurde. ${ }^{70}$ Allerdings scheint das nur eine Verkürzung der Informationswege zwischen DST und Sozialabteilung ${ }^{71}$, aber keine konspirative Kooperation zur Folge gehabt zu haben. Denn Oel war Mitglied der SPD und gehörte zu den Autoren des Sozialplans vom Sommer 1957, dessen Gesamtkonzeption einer breiten sozialen Grundsicherung für alle Staatsbürger mit den Zielen eines christdemokratisch geführten Ministeriums nicht kompatibel war.

Spätestens mit dem klaren Sieg der CDU/CSU bei den Bundestagswahlen im September 1957 war die Realisierung des Sozialplans ohnehin in weite Ferne ge-

65 Vgl. die Vermerke Referat V A 4 über die Besprechungen mit Abteilungsleiter vom 24., 26. und 30.4. sowie 6.7.1957, BAK, B 106/9789/2; Abteilungsleitervorlage vom 23.5. 1957, BAK, B 106/20652.

66 NDV 37 (1957), S. 221; vgl. ferner NDV 38 (1958), S. 34f.

67 Vgl. die Berichte über die konstituierende Sitzung der Gruppe IV „Fürsorgerechtsreform und soziale Neuordnung" am 2.5.1957, BAK, B 106/9787, sowie am 13.6.1957, LAB, B Rep. 142-9, 1263, und 16.7.1957, ADW, HGSt, SP-S XXIIIc I/O.

68 Zur Gruppe IV des DV-Studienkreises gehörten u.a.: Muthesius, Achinger, Collmer, Willi Hoppe, Heinz Keese, Franz Klein, Wyneken Kobus, Marx, Otto Ohl, Käthe Petersen, Schlegelberger und Ernst Weinbrenner.

${ }^{69} \mathrm{Vgl}$. Bertram, Staatspolitik, S. $129 \mathrm{ff}$.

70 Vgl. auch Heisig, Armenpolitik, 1990, S. 572f.

71 Vgl. etwa Vermerk Kelm vom DST, 12.1.1960, LAB, B Rep. 142-9, 1284. 
rückt. Die darin vorgesehene Konzentration der „Sozialhilfe“ auf Individualhilfen allerdings lag von der Reformlinie des Bundesinnenministeriums ohnehin nicht so weit entfernt. ${ }^{72}$ Wenn der DV-Vorstand tatsächlich gehofft hatte, daß ,in den zu erwartenden grundsätzlichen Erklärungen der neuen Bundesregierung die Fürsorgerechtsreform als dringliche Aufgabe bezeichnet" werde ${ }^{73}$, dann hatte er sich gründlich getäuscht. In seiner Regierungserklärung Ende Oktober sagte Adenauer zwar, die „Sozialreform wird fortgeführt werden“, doch die Fürsorge erwähnte er mit keinem Wort. $^{74}$

Nichtsdestoweniger hatte die Neubildung von Bundesregierung und Bundestag auch für die Fürsorge ganz unmittelbare Konsequenzen: Die Jugendabteilung wurde vom Innen- in das Familienministerium überführt und damit der eng mit der Fürsorge zusammenhängende Bereich der Jugendhilfe zum Gegenstand möglicher Ressortkonflikte. Der Bundestag legte entgegen dem Votum auch der Sozialpolitiker der Unionsfraktion den Fürsorgeausschuß mit demjenigen für Kommunalpolitik zusammen, unter Leitung von dessen Vorsitzendem Willeke, dem Generalsekretär der Kommunalpolitischen Vereinigung der CDU. ${ }^{75}$ Das minderte nicht nur das politische Gewicht der Fürsorgespezialisten unter den Ausschußmitgliedern, sondern machte den Ausschuß, der für die Beratung des Sozialhilfegesetzes zuständig sein würde, auch kommunalen Interessen zugänglicher, als es etwa die von der SPD favorisierte Zusammenlegung mit dem Sozialpolitischen Ausschuß ergeben hätte. ${ }^{76}$

Davon unabhängig erwiesen sich im Herbst 1957 auch Bemühungen um einen eigenen Fürsorgeausschuß des Bundesrates als vergeblich. Bislang wurde die Fürsorge vom Innenausschuß des Bundesrates bearbeitet, obwohl sie in den meisten Bundesländern beim Sozialminister ressortierte. Angesichts der bevorstehenden Fürsorgereform hatten sich seit Ende 1955 vor allem deren Fürsorgereferenten, unterstützt vom DST, für einen solchen Fürsorgeausschuß eingesetzt, da „Fachfragen in einem Fachausschuß schneller und gründlicher geklärt werden könnten“."77 Denn die Fürsorgereferenten der Sozialministerien waren bisher im Bundesrat auf ihr Innenministerium angewiesen und wurden häufig nicht zu den Fachberatungen des Innenausschusses hinzugezogen. ${ }^{78}$ Doch der amtierende Prä-

2 Vgl. Sozialplan, S. $116 \mathrm{ff}$.

73 NDV 37 (1957), S. 221.

74 Regierungserklärung vom 29.10.1957, in: Behn, Regierungserklärungen, S.64ff. (Zitat S. 68).

${ }^{75}$ Vgl. Niederschrift über die Sitzung des Arbeitskreises IV am 6.11.1957, ACDP, CDU/CSU-Fraktion, VIII-005-001/3, den interfraktionellen Antrag betr. Einsetzung von Ausschüssen vom 25.11.1957, BT, 3. Wp. 1957, Anlagen, Bd. 55, Drs. 18, sowie dessen einstimmige Annahme auf der Sitzung des Bundestages am 28.11.1957, BT, 3. Wp. 1957, Sten. Ber., Bd.39, S. 101.

76 Vgl. Protokoll der Sitzung der SPD-Fraktion am 30.10.1957, in: SPD-Fraktion 1957-1961, S. 9.

77 Berliner Senator für Arbeits- und Sozialwesen an den Präsidenten des Bundesrates, 14.7. 1956, Durchschrift, LAB, B Rep. 142-9, 1305; vgl. ferner DST-Hauptgeschäftsführer Ziebill an den Präsidenten des Bundesrates, 7.11.1955, ebenda.

$78 \mathrm{Vgl}$. auch für das Folgende, Kurzprotokoll der Konferenz der für das Wohlfahrtswesen zuständigen Minister am 29.5.1956, BAK, B 106/9789/2. 
sident des Bundesrates, der schleswig-holsteinische Ministerpräsident von Hassel, folgte einem Votum der Innenminister der Länder und lehnte die Bildung eines Fürsorgeausschusses aus Verfahrensgründen ab. ${ }^{79}$ Erstmals im Oktober 1957, dann wieder im Mai 1959 beantragten die Wohlfahrtsminister, zumindest im Innenausschuß einen ständigen Unterausschuß für Fürsorge zu bilden. ${ }^{80}$ Tatsächlich richtete der Ausschuß für Innere Angelegenheiten erst anläßlich der ersten Beratung des BSHG-Entwurfs von 1960 zeitlich begrenzt einen Unterausschuß „Bundessozialhilfegesetz“ ein.

In der Sozialabteilung feilte man derweil weiter am Gesetzentwurf ${ }^{81}$ : Um das tatsächliche Ausmaß der geplanten Neuerungen besser abschätzen zu können, bat Gottschick Oel im September 1957, bei „geeigneten“ Städten anzufragen, inwieweit diese schon jetzt die vorgesehenen Individualhilfen freiwillig leisteten und welche Einkommensgrenzen dabei zugrunde gelegt wurden..$^{82}$ Nachdem Oel diese Anfrage erst zwei Monate später weitergeleitet hatte, gingen die Ergebnisse der Umfrage schließlich im März 1958 beim Innenministerium ein ${ }^{83}$ : Danach erbrachten die 19 antwortenden Städte bereits fast alle der geplanten Hilfen - eine willkommene Argumentationshilfe, die sich ohne Zweifel auch der Auswahl der „geeigneten“ Städte verdankte. ${ }^{84} \mathrm{Ob}$ Scheffler allerdings tatsächlich noch glaubte, den Entwurf bis Anfang 1958 zur Kabinettsreife zu bringen, darf bezweifelt werden. Nachdem der Fürsorgetag ganz im Zeichen der Reform des Fürsorgerechts stehen sollte, mußte dieser zumindest abgewartet werden, wollte man dessen Ergebnisse berücksichtigen und die dort versammelte Fachlichkeit nicht nachträglich düpieren und damit den gewünschten Konsens gefährden.

Im DST und im DLT wurde der Fürsorgetag strategisch gründlich vorbereitet. Nachdem weder die Finanzreform 1956 noch die ersten Auswirkungen der Rentenreform die Finanzlage der Kommunen im erhofften Maße verbessert hatten, wollten beide Spitzenverbände die Reform des Fürsorgerechts eng mit einem neuen Finanzausgleich verknüpfen. Denn, so die Argumentation, jede gesetzliche Leistungsverbesserung würde ohne eine entsprechende Finanzausstattung der Kommunen zur Makulatur. Daher instruierte der DST-Arbeitskreis die städtischen Vertreter, in den einzelnen Arbeitsgruppen gleichzeitig mit der Reform des

79 Vgl. Präsident des Bundesrates an DST, 2.12.1955, sowie an den Berliner Sozialsenator, 26.7.1956, LAB, B Rep. 142-9, 1305; zum insgesamt schwierigen Verhältnis zwischen kommunalen Spitzenverbänden und Bundesrat in den fünfziger Jahren vgl. Bertram, Staatspolitik, S. $116 \mathrm{ff}$.

$80 \mathrm{Vgl}$. die Niederschriften über die Besprechung der leitenden Fürsorgereferenten der Länder am 29.7.1957 und 4.2.1958, BAK, B 106/20132, sowie über die Konferenz der für das Wohlfahrtswesen zuständigen Minister am 22.5.1959, BAK, B 106/20644.

$81 \mathrm{Vgl}$. eine überarbeitete Version des BSHG-Entwurfs vom 1.-17.10.1957, BAK, B $106 / 20648$.

82 Vgl. Gottschick an Oel am 11. 9. 1957, mit Anlage, LAB, B Rep. 142-9, 1282.

83 Gründe für die späte Weiterleitung durch Oel sind nicht ersichtlich; vgl. den Rundbrief Oels vom 14.11.1957 sowie eine tabellarische Zusammenfassung der Ergebnisse und die in einem Beiheft befindlichen Antworten der einzelnen Städte, die Oel mit Schreiben vom 20.3.1958 an Gottschick übersandte, LAB, B Rep. 142-9, 1283.

84 Oel hatte vor allem die Herkunftsstädte von Mitgliedern des DST-Sozialausschusses oder anderweitig sozial engagierter Kommunalpolitiker angeschrieben. 
Fürsorgerechts eine steuerliche Besserstellung der Kommunen zu fordern, bereitete entsprechende Entschließungsanträge vor und übersandte diese auch dem DLT. ${ }^{55}$ Wegen der starken Interdependenzen zwischen Fürsorge, Jugendhilfe und Gesundheitswesen sollte außerdem beantragt werden, auch diese beiden Bereiche gleichzeitig mit der Fürsorge zu reformieren; das hatten Muthesius und Marx schon seit längerem gefordert, sozusagen als Relikt des ursprünglich gewünschten umfassenden Sozialprogramms. ${ }^{86}$

Der Sozialausschuß des DLT verfaßte seinerseits für die Landkreisvertreter detaillierte Positionspapiere zur Argumentationshilfe jenseits reiner Obstruktion, damit „den besonderen ländlichen Verhältnissen Rechnung getragen“ werde. Je besser es auf dem Fürsorgetag gelinge, „über allgemeine sozialpolitische Theorien und Forderungen hinaus die konkreten, den einzelnen Verwaltungskörper treffenden Aufgaben zu umgrenzen, umso bessere Waffen hat der Deutsche Landkreistag in der Hand, um sich in dem jetzt wichtig werdenden Streit um die Neubegründung der kommunalen Finanzgrundlagen durchzusetzen". 87

Der Fürsorgetag vom 21. bis 23. November 1957 in Essen stand unter dem Thema „Die Neuordnung des Fürsorgerechts als Teil einer Sozialreform“. Offiziell hatte der DV-Vorstand also die Hoffnung, die Fürsorgerechtsreform sei ein integrativer Bestandteil einer umfassenden neuen Konzeption, noch nicht aufgegeben, obwohl ein belangloses Grußtelegramm aus dem Kanzleramt dies kaum mehr rechtfertigte. ${ }^{88}$ Scheffler jedenfalls zog in seinem einleitenden Vortrag „den Rahmen der Betrachtung [...] wesentlich enger" 89 und lüftete nun offiziell den „dichte[n] Schleier über der erhofften Fürsorgerechtsreform “90, indem er ausführlich und konkret über die wesentlichen Inhalte und Ziele des Entwurfs seiner Abteilung referierte. Neben dem Bestreben, im sich wandelnden sozialpolitischen Klima den ungebrochenen Modernisierungswillen des Bundesinnenministeriums zu illustrieren ${ }^{91}$, wollte er, wenn er etwa bereits DM-Beträge für mögliche Einkommensgrenzen nannte, auch sein eigenes Haus auf den erreichten Arbeitsstand öffentlich festlegen. Scheffler würde nämlich ein Jahr früher als geplant aus Gesundheitsgründen Ende Januar 1958 in den Ruhestand treten und konnte daher den Gesetzentwurf nicht mehr selbst fertigstellen. ${ }^{92}$

Auch die sieben Arbeitsgruppen des Fürsorgetags gingen medias in res und diskutierten für alle relevanten Themenbereiche konkrete Forderungen für das ge-

85 Vgl. die Niederschriften über die Sitzungen des DST-Arbeitskreises „Fürsorgerecht“ am 7. und 16.11.1957, LAB, B Rep. 142-9, 1282.

86 Vgl. Niederschrift über die Sitzung des Arbeitskreises am 7./8. 5. 1957, ebenda.

87 Niederschrift über die Sitzung des DLT-Sozialausschusses vom 8.11.1957, mit Anlagen, BAK, B 172/444-1/1.

88 Das knappe Telegramm Adenauers betonte nur floskelhaft die „hohe menschliche Verpflichtung" der Fürsorge und die Wichtigkeit der Beratungen des Fürsorgetags, erwähnte aber die geplante Reform des Fürsorgerechts nicht; vgl. Neuordnung des Fürsorgerechts, S. 10.

89 Gerhard Scheffler, Neuordnung, S. $20 f$.

90 Vgl. BldW 105 (1958), S. 1.

91 Vgl. auch ebenda, S. 3.

92 Vgl. ebenda, S. 72. 
plante Bundesfürsorgegesetz. Während sie meist die von den jeweiligen DV-Ausschüssen vorbereiteten Leitsätze, z.T. modifiziert, verabschiedeten, kam die mit der Rechtsstellung des Hilfeempfängers und der Trägerfrage befaßte Gruppe wegen heftiger Auseinandersetzungen über die Rolle der freien Wohlfahrtspflege zu keiner Einigung. Auch die mit der Jugendhilfe befaßte Arbeitsgruppe entglitt der Regie des DV-Vorstands und forderte eine Herauslösung aller Hilfen für Minderjährige aus dem Fürsorgerecht. Allerdings gelang dem DST-Vertreter hier wie in der von Marx geleiteten Arbeitsgruppe zur Gesundheitsfürsorge, die im DST vorbereiteten Entschließungen tatsächlich auch zur Verabschiedung zu bringen und so die fachliche Autorität des DV für die kommunalen Interessen einmal mehr zu nutzen..$^{93}$

Inwieweit die konkreten Ergebnisse des Fürsorgetages, wie vom neuen Staatssekretär Georg Anders versprochen, bei den weiteren Arbeiten seines Hauses „besondere Beachtung" fanden ${ }^{94}$, ist nicht generell zu bemessen. Sofern sie den bisherigen Planungen Schefflers und Gottschicks, wie etwa bei den Hilfen für Jugendliche, deutlich zuwiderliefen oder aber, wie bei der Hauspflege, erheblich höhere Leistungen beinhalteten, wurden sie nicht berücksichtigt. Wenn sie aber, wie bei der Hilfe für Gefährdete, ohnehin auf der Linie des Ministeriums lagen, wurden sie auch bis ins Detail übernommen. Schließlich war der Fürsorgetag keine neue, völlig von den bisherigen Reformgremien abgekoppelte Instanz, sondern vielfältig mit diesen verwoben und über Leiter und Referenten in den einzelnen Arbeitsgruppen auch durch diese beeinflußt. In vieler Hinsicht war der Fürsorgetag daher eher eine öffentliche Plattform zur fachlichen Legitimierung der Reformarbeit im Bundesinnenministerium, als daß er umgekehrt diese maßgeblich geprägt hätte.

Ein Ende November 1957 fertiggestellter neuer Referentenentwurf jedenfalls zeigte bei Einzelregelungen eher Spuren der Beratungen mit Marx und Muthesius und der Diskussionen in dem Beirats-Fürsorgeausschuß, dem DST-Arbeitskreis und der Untergruppe des DV-Studienkreises, ohne die Grundrichtung der Reformarbeit geändert zu haben. ${ }^{95} \mathrm{Neu}$ war jetzt, daß auch die Blindenhilfe als eigene Hilfeart eingeführt und das Körperbehindertengesetz (KBG) und das noch im Entwurf befindliche Tuberkulosehilfegesetz (THG) eingearbeitet worden waren. Nachdem sowohl die anderen Referate der Sozialabteilung als auch Marx und Muthesius grünes Licht gegeben hatten, wurde der Entwurf Anfang Januar 1958 den betroffenen Nachbarreferaten und -abteilungen zur Stellungnahme übersandt ${ }^{96}$, und der Bundesinnenminister kündigte den Bundestagsausschüssen für Kommunalpolitik und Fürsorge sowie Inneres im Rahmen seines Gesetzgebungs-

93 Vgl. Neuordnung des Fürsorgerechts, S. 96ff., 269ff., 421, 435; allgemein Jaedicke u.a., Politik, S. 45f.

94 Neuordnung des Fürsorgerechts, S. 18.

95 Vgl. den BSHG-Entwurf vom 29.11.1957, BAK, B 106/20643.

96 Vgl. Scheffler an Marx/Muthesius, 30.11.1957; Referat V A 4 an Referate A 1 etc. der Abteilung V, 3. 12.1957; Vermerk Referat V A 4 über Besprechung mit Marx und Muthesius vom 12.12.1957; Referat V B 1 an Referat V A 4, 13.12.1957; Vermerk V A 4 über die Besprechung mit den Referaten V A 1 etc. vom 18.12.1957; Referat V A 4 an die Referate I A 3 etc., 9.1.1958, ebenda. 
programms das Bundessozialhilfegesetz an. ${ }^{97}$ Die Unterabteilung für Kommunalwesen kritisierte zwar grundsätzlich die „perfektionistische Ausgestaltung“ des Entwurfs, wünschte aber konkret nur Änderungen bei einzelnen Verfahrensvorschriften. ${ }^{98}$ Die Gesundheitsabteilung hingegen lehnte den geplanten Einbau des KBG und des THG rundheraus ab: Allgemeines Gesundheitsrecht gehöre nicht in ein Fürsorgegesetz. Demgegenüber beharrte die Sozialabteilung auf ihrem Ziel, das gesamte Fürsorgerecht zusammenzufassen und keine fürsorgerischen Einzelgesetze mehr zuzulassen. Es bedurfte eines halben Jahres zäher Verhandlungen, ehe die Gesundheitsspezialisten ihren Widerstand aufgaben und der Einarbeitung zustimmten. ${ }^{99}$

Doch noch ein weiterer Grund verzögerte die Fertigstellung des ersten offiziellen Referentenentwurfs: Nachdem Scheffler Ende Januar 1958 pensioniert worden war, trat gut anderthalb Monate später mit Johannes Duntze ein neuer Abteilungsleiter den Dienst an, der sich erst mit den Reformarbeiten vertraut machen wollte. ${ }^{100}$ Der badische Verwaltungsjurist hatte sich als Spezialist für Flüchtlingsfragen einen Namen gemacht und war seit 1951 im Stuttgarter Ministerium für Vertriebene, Flüchtlinge und Kriegsgeschädigte Stellvertreter des Ministers. ${ }^{101}$ Außerdem, und das war für einen wichtigen Teilaspekt der weiteren Gesetzesgenese von Bedeutung, war er Mitglied des Vorstandes des Central-Ausschusses für die Innere Mission und damit ähnlich wie sein Referent für Allgemeine Fürsorge und Fürsorgerische Sonderaufgaben, Arnold Weller, der evangelischen Diakonie eng verbunden. ${ }^{102}$ Duntze war offenbar gewillt, die Reformlinie seines Vorgängers fortzusetzen; doch sein Vorgehen vermittelt bisweilen den Eindruck größerer Nachgiebigkeit gegenüber Kritikern. Ob Scheffler die Aufweichung der ursprünglich rigideren bzw. großzügigeren Planungen des Bundesinnenministeriums 1958/59 allerdings hätte verhindern können, ob also insofern der Wechsel an der Spitze der Sozialabteilung erhebliche Konsequenzen für die Fürsorgereform hatte, ist angesichts der politisch gewichtigen Widerstände gegen diese Planungen zu bezweifeln.

Mittlerweile hatten auch DST und DLT offiziell Position bezogen: Nach dem Fürsorgetag beriet der DST-Sozialausschuß im Dezember die Vorlage seines Reform-Arbeitskreises zum materiellen Fürsorgerecht, die weitestgehend der Linie des Innenministeriums entsprach bzw. in einigen Punkten sogar auf Bitten Gottschicks noch auf diese Linie gebracht wurde, um die Fertigstellung des Referen-

97 Vgl. Abteilungsleitervorlage vom 17.1.1958, BAK, B 106/9697.

98 Unterabteilung I C an Abteilung V, 19.3.1958, BAK, B 106/20643.

99 Vgl. die Vermerke Referat V A 4 betr. Beteiligung der Abteilung IV vom 18.2. und 5.3. 1958, ebenda.

$100 \mathrm{Vgl}$. auch einen vertraulichen internen Bericht des DLT vom Mai 1958, BAK, B 172/444-01/1.

101 Vgl. BldW 105 (1958), S. 132; NDV 38 (1958), S. 139; Hammerschmidt, Wohlfahrtsverbände in der Nachkriegszeit, S.419f. Duntze (1901-1987) leitete die BMI-Sozialabteilung bis 1966.

102 Vgl. Taschenbuch der Evangelischen Kirchen (1955), Bd.I, S.148; Arnold Weller war 1946-1949 beim Evangelischen Hilfswerk tätig gewesen; vgl. Heisig, Armenpolitik, 1990, S. 597. 
tenentwurfs nicht weiter zu verzögern. Trotz erheblicher Widerstände des konservativen Bayerischen Städteverbands wurde diese Stellungnahme von Präsidium und Hauptausschuß des DST Ende Februar 1958 verabschiedet und Mitte April dem Ministerium übersandt. ${ }^{103}$ Ein Artikel Oels in der April-Nummer der Verbandszeitschrift sollte auch die Mitgliedsstädte von dieser Position überzeugen und unter Verweis auf die Umfrage über die bereits erbrachten freiwilligen Leistungen gerade die Stadtkämmerer beruhigen. ${ }^{104}$ Insgesamt hatten sich damit die Fürsorgeexperten des DST als eine der wirksamsten Stützen der Reformarbeit des Bundesinnenministeriums erwiesen.

Ganz anders der DLT: Offensichtlich war man hier doch von dem von Scheffler avisierten Ausmaß der Reform überrascht. Jedenfalls warnte Bangert im JanuarHeft des Verbandsblatts vor einer „hemmungslosen Kasuistik“ und kritisierte die einheitlichen Leistungsstandards, die Höhe der Einkommensgrenzen und die geplante Einschränkung der Kostenersatzpflicht; unter Berufung auf das Subsidiaritätsprinzip forderte er jetzt, die Fürsorge erst nach Abschluß der geplanten Reformen der Krankenversicherung und der Versorgungsgesetze, gleichzeitig mit der Finanzverfassung neu zu ordnen. ${ }^{105}$

Der Fürsorgeausschuß des Beirats geriet zunehmend unter Zeitdruck, wenn er noch vor Fertigstellung des offiziellen Referentenentwurfs eigene Vorschläge machen wollte. Er erwies sich dabei immer mehr als Gremium, das nicht, wie etwa im Rückblick eines Beteiligten aus dem Bundesarbeitsministerium behauptet, die Reform der Fürsorge vorbereitete ${ }^{106}$, sondern vor allem den Arbeitsstand des Innenministeriums nachbearbeitete. Der Ausschuß, an dessen Sitzungen im Frühling kaum mehr als fünf oder sechs Mitglieder teilnahmen, beriet seit dem Fürsorgetag die einzelnen Fragepunkte des von Scheffler seinerzeit vorgelegten Katalogs relativ zügig und gewährte dabei, wenn auch nicht in allen Einzelheiten deckungsgleich, den Plänen der Sozialabteilung zusätzliche fachlich-neutrale Legitimität. ${ }^{107}$

103 Vgl. Niederschrift über die Sitzung des DST-Sozialausschusses am 13./14.12.1957 (Auszug), mit Vorbericht zu Punkt 2 der Tagesordnung vom 9.12.1957; Bayerischer Städteverband an Ziebill, 9.12.1957; Vorbericht für die 85. Sitzung des DST-Präsidiums vom 18.2.1958; Niederschrift über dessen Sitzung (Auszug) am 28.2.1958, LAB, B Rep. 142-9, 1282; Niederschrift über die Sitzung des DST-Hauptausschusses (Auszug) am 28.2./1.3.1958, Stellungnahme des DST zur Neuregelung des Fürsorgerechts vom 1.3.1958 sowie DST an Abteilung V bzw. „Kommunalabteilung“ des BMI, 14.4.1958, LAB, B Rep. 142-9, 1283. Der zweite Teil zum formalen Fürsorgerecht, der ebenfalls vom Arbeitskreis und dem DST-Sozialausschuß vorbereitet worden war, wurde erst nach dem ersten Referentenentwurf vom Juli 1958 fertig; vgl. Niederschrift über die Sitzung des DST-Sozialausschusses (Auszug) am 20./21.6.1958, mit Stellungnahme zur Neuregelung des Fürsorgerechts (II. Teil) vom 20.8.1958, ebenda.

104 Vgl. Oel, Neuordnung des Fürsorgerechts.

105 Vgl. Die Selbstverwaltung 12 (1958), S. 9 ff.

106 Vgl. Zöllner, Jahrhundert, S.148; so dann auch Tennstedt, Fürsorgegeschichte, S. 100; ähnlich Hockerts, Ausblick, S. 260; Schmitt, Arbeits- und Sozialverwaltung, S. 573.

107 Vgl. die Niederschriften über die Sitzungen des Arbeitsausschusses für Fragen der Fürsorge am 4.12.1957, 30./31.1., 21./22.2., 21./22.3., 10./11.4., 25.4., 6./7.6. sowie 27./28.6.1958, ADW, HGSt 6769. Die Gruppe IV des DV-Studienkreises trat nach dem Fürsorgetag nicht mehr zusammen. 
In einer neuen, nun erstmals von Duntze verantworteten Version vom April 1958 war der Sozialhilfegesetzentwurf redaktionell überarbeitet und mit Rücksicht auf die Gesundheitsabteilung der besondere Charakter der Regelungen für Körperbehinderte stärker herausgestrichen worden. Darüber hinaus wurden einzelne Bestimmungen zugunsten der Hilfeempfänger verschärft (Regelsätze) und weiter ausgebaut (Ausbildungshilfe), andere zugunsten der Träger gelockert (Arbeitsfürsorge, Hauspflege, Personal). Auch Duntze informierte Marx und Muthesius streng vertraulich über den Entwurf. ${ }^{108}$ Anfang Juni verkündete Duntze im Fürsorgeausschuß des Beirats, daß der Gesetzentwurf „in nächster Zeit den Bundesressorts und weiteren Beteiligten zur Stellungnahme zugeleitet" werden und bis Jahresende kabinettsreif sein solle. ${ }^{109}$ Muthesius drängte daher auf schnellen Abschluß der Beratungen und Veröffentlichung der Empfehlungen. Ende Juni erledigte der Ausschuß daraufhin in seiner zwanzigsten und letzten Sitzung die noch offenen Punkte, und Theodor Marx übernahm die redaktionelle Überarbeitung der Empfehlungen zur Vorlage an den Beirat. ${ }^{110}$ Dieser tagte jedoch erst wieder im Oktober, so daß die Empfehlungen, wie man im Arbeitsministerium etwas verärgert registrierte, erst nach Herausgabe des ersten Referentenentwurfs veröffentlicht werden konnten. Wie erwartet, stützten sie weitestgehend den Entwurf des Innenministeriums und verliehen diesem damit eine positive Expertise. ${ }^{111}$

Im Referentenentwurf vom Juli 1958 waren zum Bedauern Schefflers unter seinem Nachfolger nicht nur die Einkommensgrenzen für die „Hilfen in besonderen Lebenslagen“ deutlich herabgesetzt (s.u.), sondern auch solche Formulierungen gestrichen worden, die zwar sicherlich „das Gesetz vom legislatorischen Standpunkt gesehen nicht verbesserten“, die man aber ursprünglich aufgenommen hatte, „um die Praxis in Richtung einer möglichst einheitlichen Anwendung der Vorschriften und auch im Interesse des Hilfesuchenden zu binden“.112 In $138 \mathrm{~Pa}$ ragraphen wurde jetzt die gesamte bisher auf zahlreiche Verordnungen, Sonder-

108 Vgl. Duntze an Marx/Muthesius, 28.4.1958, BAK, B 106/20643; Gottschick an Marx, 29.5.1958, BAK, B 106/9789/1.

109 Niederschrift über die Sitzung des Arbeitsausschusses am 6./7.6.1958, ADW, HGSt 6769.

110 Vgl. Niederschrift über die Sitzung des Arbeitsausschusses am 28./29.6.1958, ebenda.

111 Theodor Marx war am 3. 7.1958 tödlich verunglückt, vgl. NDV 38 (1958), S. 201ff.; Heisig, Armenpolitik, 1990, S. 565f., hatte aber vor seinem Tod die Vorlage noch weitgehend fertiggestellt, vgl. einen Vermerk Bangerts über ein Gespräch mit Detlev Zöllner vom BMA vom 4. 8.1958, BAK, B 172/444-01/6. Im Beirat lehnten es die Vertreter der SPD (u.a. Auerbach) und des DGB ab, die umfangreiche Vorlage des Fürsorgeausschusses ohne Aussprache zur Veröffentlichung freizugeben und damit letztlich den Beirat selbst seiner legitimen Funktion zu berauben; Arbeitsminister Blank und Muthesius konnten sich jedoch mit ihrem Wunsch nach schnellstmöglicher Veröffentlichung durchsetzen; vgl. Niederschrift über die Sitzung des Beirats für die Neuordnung der sozialen Leistungen am 13.10.1958, ADW, HGSt 6769; ferner einen wohl von Zöllner stammenden Beitrag über die Empfehlungen des Ausschusses in: SF 7 (1958), S.248f. Die Empfehlungen des Arbeitsausschusses für Fragen der Fürsorge wurden veröffentlicht u.a. in: NDV 38 (1958), S. 301ff.

112 Scheffler an Duntze, 8. 8.1958, BAK, B 106/20643; vgl. Entwurf eines Gesetzes über die Gewährung von Sozialhilfe vom Juli 1958, im Folgenden: BSHG-Entwurf 7/1958, BAK, B 106/20643. 
gesetze und Verwaltungsvorschriften verteilte Materie des Fürsorgerechts zusammengefaßt und neu geregelt. Auf einen einleitenden Grundsatzteil folgte ein Abschnitt über die „Hilfe zum Lebensunterhalt“, also über die traditionelle laufende Unterstützung einschließlich der „Hilfe zur Arbeit“; der umfangreichste war der dritte Abschnitt „Hilfe in besonderen Lebenslagen“; weitere Abschnitte behandelten das formale Sozialhilferecht wie wirtschaftliche Voraussetzungen der Hilfegewährung, Trägerschaft etc. Der Entwurf, so Gottschick vor dem Hauptausschuß des DV, nehme "weitgehend die in der Praxis und in Sonderbestimmungen schon sichtbar gewordene Weiterentwicklung der Fürsorge auf und versucht gleichzeitig, den Blick in die Zukunft frei zu machen, indem er seinerseits die Möglichkeit auch für eine künftige Weiterentwicklung [...] bietet“. Das Gesetz bringe daher „keine revolutionäre, sondern eine evolutionäre Lösung“. 113

Die allgemeine Begründung zu dem Entwurf enthüllte allerdings auch dessen Achillesferse: Angaben über die entstehenden Mehrkosten seien „nicht, auch nicht schätzungsweise, möglich". ${ }^{114}$ Spätestens jetzt wurde ein wesentliches Manko der gesamten Fürsorgereform deutlich: Der Arbeit der Sozialabteilung wie der Reformgremien hatte kein „umfassendes empirisches (und auf seine strukturelle Relevanz geprüftes) Material über die Funktion der verschiedenen Sozialhilfearten in der gegenwärtigen westdeutschen Gesellschaft" zugrunde gelegen ${ }^{115}$; diesen Mangel konnten keine Einzelgespräche mit Praktikern und Fürsorgefunktionären oder Umfragen unter knapp zwanzig kreisfreien Städten wettmachen. Das Fehlen fundierter Angaben über mögliche Empfängerzahlen und Kosten würde Reformskeptikern die Argumentation erleichtern und zur Verunsicherung der Beamtem des Innenministeriums beitragen.

Unter dem 25. Juli 1958 verschickte Duntze den offiziellen ersten Referentenentwurf eines „Gesetzes über die Gewährung von Sozialhilfe (Bundessozialhilfegesetz - BSHG)“ in jeweils zahlreichen Exemplaren an alle einschlägigen Stellen mit der Bitte um Stellungnahme: die betroffenen Ministerien des Bundes und der Länder, die kommunalen Spitzenverbände, den DV, die Arbeitsgemeinschaft der Landesfürsorgeverbände, die Spitzenverbände der freien Wohlfahrtspflege, die Zentrale Fürsorge-Spruchstelle, den DGB, die Arbeitsgemeinschaft für Jugendpflege und Jugendfürsorge (AGJJ), die Behindertenverbände und die Organisationen der Sozialarbeiter. Außerdem erhielten einzelne Fürsorgeexperten und Abgeordnete der CDU, der Innenausschuß des Bundesrates und die Bonner Vertretungen der beiden Kirchen den Entwurf zur Information. ${ }^{116}$ Duntze hatte zwar die Adressaten darum gebeten, den Entwurf noch nicht öffentlich zu diskutieren; wie angesichts der breiten Streuung kaum anders zu erwarten, sickerten jedoch Details in die Öffentlichkeit: Am 14. August meldete die „Frankfurter Allgemeine Zeitung" die Fertigstellung des Referentenentwurfs, und kurz darauf erschien mit

113 Gottschick, Referentenentwurf, S. 16.

114 Vgl. Bemerkungen zum BSHG-Entwurf 7/1958, S. 24, BAK, B 106/20643.

115 So Matthes, Konzeptionenstreit, S. 13, vor allem mit Blick auf die im BSHG getroffenen Regelungen zur Stellung der freien Wohlfahrtspflege, vgl. auch S. 83, ebenda.

116 Vgl. die Durchschläge der betreffenden Schreiben Duntzes vom 25.7.1958, eine erste, undatierte Versandliste sowie eine Liste vom 23.9.1958, BAK, B 106/20643. 
Duntzes Zustimmung auch eine kurze Notiz im Nachrichtendienst des DV. ${ }^{117}$ Im Oktober-Heft der „Blätter der Wohlfahrtspflege“, einer der wichtigsten Fachzeitschriften, folgte eine zustimmende und ausführliche, einzelne Paragraphen sogar wörtlich zitierende Besprechung des „umfangreichen und kühnen Gesetzentwurfs" durch die sozialdemokratische Fürsorgeexpertin des hessischen Sozialministeriums Käte Pluskat. ${ }^{118}$ Daraufhin baten im Laufe des Herbstes weitere interessierte Stellen und vor allem Abgeordnete um die Zusendung des Referentenentwurfs, keineswegs immer mit Erfolg. Neben begründetem sozialpolitischem Informationsbedarf war offensichtlich vor allem die vermutete Nähe zur Reformlinie des Bundesinnenministeriums das entscheidende Kriterium für die Weitergabe: Während Gottschick auch andere Sozialpolitikexperten der CDU-Bundestagsfraktion oder die Bundesvereinigung der deutschen Arbeitgeberverbände anstandslos mit dem Entwurf versorgte, mußte etwa der führende SPD-Experte Willy Könen vom Bundestagsausschuß für Kommunalpolitik und öffentliche Fürsorge erst nachfragen, und selbst die CSU erhielt nur nach persönlicher Intervention des Vorsitzenden der Landesgruppe Hermann Höcherl bei Schröder die nötigen Exemplare. ${ }^{119}$ Wahrscheinlich fürchtete die Sozialabteilung angesichts der bekannten Ressentiments der bayerischen Kommunen gegen die Fürsorgereform frühzeitige Interessenkoalitionen mit den bayerischen Unionsabgeordneten. Auch beim Bundestagsausschuß vertraute man vor allem auf alte Verbündete: Während Duntze und Gottschick einerseits überlegten, ob der Vorsitzende Willeke überhaupt ins Bild gesetzt werden sollte, überließen sie seiner Stellvertreterin und ehemaligen Vorsitzenden des alten Fürsorgeausschusses Maria Niggemeyer (CDU) Anfang 1959 sogar streng vertraulich sämtliche eingetroffenen Stellungnahmen. ${ }^{120}$

Diese Informationspolitik war aber nicht allein wegen dieser Auswahl bemerkenswert. Zwar erklärte Gottschick vor dem DV-Hauptausschuß, es sei von seinem Hause von Anfang an geplant gewesen, „nicht wie aus heiterem Himmel mit einem Gesetzentwurf herauszukommen, der mangels notwendiger zeitlicher Voraussetzungen nicht hätte durchgearbeitet sein können“121; und auch die Einschaltung der kommunalen Spitzenverbände noch im Stadium des Referentenentwurfs widersprach keineswegs den üblichen Gepflogenheiten der Ministerialbürokratie. ${ }^{122}$ Eine derart großzügige Verteilung nicht nur von Informationen, sondern auch der Bitte um Stellungnahme war allerdings mit der Geschäftsordnung der Bundesregierung kaum vereinbar und stieß im Kabinett offensichtlich auf Kri-

117 Vgl. Frankfurter Allgemeine Zeitung vom 14.8.1958; Vermerk Duntze vom 14. 8.1958, Duntze an Muthesius, 15.8.1958, Muthesius an Duntze, 18.8.1958, BAK, B 106/20643; NDV 38 (1958), S. 229.

118 Pluskat, Gedanken, S. 313.

119 Vgl. die Versandlisten zum BSHG-Entwurf 7/1958 (o.D. bzw. vom 23.9.1958), ferner Gottschick an Niggemeyer am 5.9.1958 und Peter Horn am 6.10.1958, sowie den Briefwechsel zwischen Helmut Geiger von der CSU-Landesgruppe und Referaten der Sozialabteilung am 2. und 5.9.1958, sowie zwischen Höcherl und Schröder am 15. und 25. 9. 1958, BAK, B 106/20643.

120 Vgl. Gottschick an Niggemeyer am 5. 9.1958, ebenda, und 2.2.1959, BAK, B 106/20644.

121 Gottschick, Referentenentwurf, S. 16.

122 Vgl. Bertram, Staatspolitik, S. 115f., 124; Voigt, Partizipation, S.66ff. 
tik. ${ }^{123}$ Über die Gründe von Duntzes Vorgehen ist nichts bekannt. Vermutlich waren es die gleichen, die auch seinen Vorgänger Scheffler zur frühzeitigen Einbeziehung der verschiedensten Experten und Gremien veranlaßt hatten: die Suche nach Konsens mit den ausführenden Stellen und nach fachlicher Rückendeckung für den künftigen Regierungsentwurf.

$\mathrm{Daß}$ diese Rückendeckung selbst innerhalb der Regierungspartei immer nötiger werden würde, war auf dem Parteitag der CDU vom 19. bis 21. September 1958 in Kiel deutlich geworden: In einer aufsehenerregenden Rede hatte Gerstenmaier abermals erklärt, daß „,in allem Wesentlichen die Grenzen des sozialen Rechtsstaates nach der Leistungsseite erreicht" seien, da trotz eines ausgebauten Sozialsystems die Ansprüche an den Staat weiter wüchsen, während die individuelle Leistungsbereitschaft sinke. Sonst drohe die „Gefahr, daß wir kopfüber in das Gesellschaftskonzept des modernen Sozialismus stürzen“, der „durch die weitere Ausdehnung der Staatskompetenzen die egalitäre Massengesellschaft" organisiere, den einzelnen immer stärker kontrolliere und seiner Freiheit beraube. ${ }^{124}$ Unter Berufung auf liberales Gedankengut ebenso wie auf das Subsidiaritätsprinzip der katholischen Soziallehre betonte er den Primat der Pflicht zur Selbsthilfe und erklärte, daß es nicht Aufgabe des sozialen Rechtsstaates sein könne, seinen Bürgern ohne Rücksicht auf deren Vorleistungen und Leistungsvermögen „das letzte Risiko der wirtschaftlichen, der sozialen Existenz ab[zu]nehmen“. ${ }^{125}$ Die Stoßrichtung dieser Rede wurde zwar noch in Kiel von namhaften Vertretern des Arbeitnehmerflügels der Union unter Verweis auf das ebenso in der katholischen Soziallehre verankerte Prinzip der Solidarität kritisiert und blieb auch weiterhin umstritten. ${ }^{126}$ Sie war aber ein deutliches Zeichen für die wachsenden sozialpolitischen Divergenzen innerhalb der Regierungspartei, die eine zumindest großzügige, wenn auch nur „partielle Komplettierung“127 der „Sozialreform“ zunehmend erschwerten. ${ }^{128} \mathrm{Da}$

${ }^{123}$ Vgl. einen Vermerk der DLT-Referentin Wolff vom 16.4.1959, BAK, B 172/444-2/2; ferner allgemein die Sondersitzung des Bundeskabinetts am 28.5.1958, wo Adenauer „seinen bereits mehrfach geäußerten Wunsch“ wiederholte, „daß die Ressorts eine grundsätzliche Stellungnahme des Kabinetts herbeiführen, bevor Gesetzentwürfe oder sonstige Maßnahmen von besonderer politischer Bedeutung mit den Ländern oder mit Fachkreisen erörtert werden“, Kabinettsprotokolle 1958, S. 262. Grundsätzlich überließ es die Geschäftsordnung der Bundesministerien dem Ermessen des zuständigen Ressortministers, ob und inwieweit er außer den Landesministerien auch die beteiligten Fachkreise oder auch Abgeordnete, die Presse und amtlich nicht beteiligte Personen bei der Erstellung eines Entwurfs hinzuzog; vgl. \$S 23-25 der Gemeinsamen Geschäftsordnung der Bundesministerien. Besonderer Teil (GGO II) in der noch nicht vom Kabinett verabschiedeten, aber seinerzeit in der Praxis getesteten Fassung, veröffentlicht bei Lechner/Hülshoff, Parlament, S.399ff. Laut $\mathbb{} 23$ Abs. 2 GGO II war allerdings bei „Gesetzentwürfen von besonderer politischer Bedeutung“ zuvor eine Grundsatzentscheidung des Bundeskabinetts einzuholen und im übrigen „darauf zu achten, daß mit den Vertretungen der Fachkreise nicht in einer Weise Fühlung genommen wird, die dem Kabinett die Entscheidung erschwert".

124 Eugen Gerstenmaier, „Staatsordnung und Gesellschaftsbild“, in: Christlich Demokratische Union Deutschlands. 8. Bundesparteitag, S. 90ff., Zitat S. 98.

125 Ebenda, S. 95, vgl. ferner S. $102 f$.

126 Vgl. die Beiträge von Josef Arndgen und Hans Katzer ebenda, S. 128ff., $143 \mathrm{ff}$.

127 Zöllner, Sozialpolitik, S. 376.

128 Vgl. Hockerts, Ausblick, S. 257f. 
mochte die Einschätzung des Darmstädter Oberbürgermeisters Engel auf der kommunalpolitischen Bundeskonferenz der SPD Mitte September, mit dem Referentenentwurf zum Bundesfürsorgegesetz hätten „sich beim Bundesgesetzgeber sozialdemokratische Forderungen im wesentlichen durchgesetzt“, wenig hilfreich sein. ${ }^{129}$ Ein auf dem CDU-Parteitag zusammengestelltes Arbeitsprogramm über die wichtigsten innenpolitischen Aufgaben erwähnte zwar u.a. die Reform der Unfall- und der Krankenversicherung, ein Berufsausbildungsgesetz und eine Verbesserung des Kindergeldes, die Fürsorgereform hingegen - wieder einmal - nicht. ${ }^{130}$ Das Bundeskabinett hingegen hatte am 3. Juni 1958 das Bundessozialhilfegesetz in eine Liste zentraler Gesetzesvorhaben aufgenommen ${ }^{131}$, die möglichst Anfang 1959 kabinettsreif sein sollten, damit sie nicht unter Zeitdruck verabschiedet werden müßten; auf eine Anfrage des Bundeskanzleramts hin sicherte Gottschick Anfang Oktober diesen Termin auch zu. ${ }^{132}$

Während in den Verbänden über den Entwurf beraten wurde und erste Ressortbesprechungen stattfanden, stellten Duntze und Gottschick diesen am 18. Oktober auch vor dem Hauptausschuß des DV vor. Die „extensive Sozialpolitik“ der vergangenen Jahre, so Duntze, mit ihrer immer weiter ausgebauten Absicherung der gängigen Lebensrisiken bedürfe nun der „Intensivierung“ durch die Fürsorge; diese müsse sich vor allem denjenigen zuwenden, „die infolge der Besonderheit ihrer Lebenslage von Arbeit und Verdienst, von sinnvoller Gestaltung ihres Lebens ausgeschlossen sind. Ihnen die Wege zum sinnerfüllten Leben zu weisen und zu ebnen“, werde die künftige Aufgabe einer sozialpädagogisch orientierten Fürsorge sein. ${ }^{133} \mathrm{Ob}$ sich dieser optimistische Ansatz angesichts erster sehr unterschiedlicher Reaktionen im Hauptausschuß würde halten lassen, mußte sich allerdings erst noch zeigen. ${ }^{134}$

Bei den für die weitere Arbeit besonders wichtigen Besprechungen mit den leitenden Fürsorgereferenten der Länder im Oktober und November jedenfalls ließ der Entwurf bereits die von der hessischen Vertreterin Pluskat befürchteten „ersten Federn“. 135 Die Ländervertreter, die sich zum Teil über die Haltung ihrer regionalen kommunalen Spitzenverbände informiert hatten, beurteilten den Entwurf zwar insgesamt positiv, hielten ihn aber mehrheitlich für zu weitgehend und konnten sich gegenüber den Planern im Innenministerium mit dem Wunsch durchsetzen, Rechtsansprüche und Einkommensgrenzen teilweise zu lockern und Vorgaben über die Durchführung der Hilfen zu streichen. ${ }^{136}$ Wenn auch die mei-

129 Referat Engel auf der SPD-Konferenz [am 12./13. 9.1958], Anlage zu Ziebill an Oel am 15. 9.1958, Ms., Auszug, LAB, B Rep. 142-9, 1283.

130 Das Arbeitsprogramm ist abgedruckt in: Richter, Sozialreform, Bd. 6 G I.

131 Vgl. Kabinettsprotokolle 1958, S. $263 \mathrm{ff}$.

132 Vgl. Referat I A 2 an die Abteilungsleiter II etc., 7.10.1958, sowie Referat V A 4 an Referat I A 2, 9. 10.1958, BAK, B 106/20643.

133 Duntze, Referentenentwurf, S. 15.

134 Vgl. NDV 39 (1959), S. 23f.; Vermerk Referat V 4 vom 23. 10.1958, BAK, B 106/9786/1.

135 Vgl. Pluskat, Gedanken, S. 313.

136 Vgl. dazu die Ergebnisniederschrift über eine [interne Vor-]Besprechung der leitenden Fürsorgereferenten der Länder am 6./7.10.1958, LAS Abt. 761 Nr. 11084; Niederschriften über die Besprechungen mit Vertretern der obersten Landessozialbehörden am 21./22. 10. bzw. 6.11.1958, LAS Abt. 761 Nr. 8874. Zu den Kontakten zwischen Mini- 
sten Länderspezialisten einem Ausbau der Fürsorge aufgeschlossen gegenüberstanden, so wachten doch auch sie darüber, daß der Bund die Verwaltungs- und Finanzhoheit der Länder nicht beeinträchtigte und gerade im primär der Länderkompetenz zuzurechnenden Bereich der Fürsorge diesen genügend Gestaltungsund Finanzierungsspielräume ließ.137

Die Stellungnahme des DST - ebenfalls von zentraler Bedeutung, da hier ein Teil der eigentlichen Sozialhilfeträger zu Worte kam - wies in eine ähnliche Richtung: Geschäftsführer Ziebill konstatierte zwar eine „weitgehende(r) Berücksichtigung der Auffassungen und Wünsche des Deutschen Städtetages im vorliegenden Entwurf“138, doch auch der DST beklagte „eine Tendenz zum Perfektionismus" und umständliche, schwierig anzuwendende Formulierungen. Der DST wünschte daher die Streichung von „Anweisungen über das administrative Vorgehen der Träger" oder „rein programmatische[r] Bestimmungen“; außerdem müsse man auf die „Grenze des finanziell Möglichen gerade beim vorliegenden Gesetz mit aller Deutlichkeit hinweisen“. ${ }^{139}$

Demgegenüber fiel die Kritik des traditionell ja unionsnahen Landkreistages geradezu vernichtend aus: DLT-Hauptgeschäftsführer Wormit bezeichnete das geplante Gesetz vor schleswig-holsteinischen Landräten als „Albdruck“140, und die offizielle Stellungnahme des DLT-Sozialausschusses verurteilte die „allgemeine sozialpolitische Tendenz des Entwurfs“, sei er doch „ein weiterer Schritt in jenen Wohlfahrts- und Versorgungsstaat [...], der die soziale Selbstverantwortung des Individuums wie der Familie mißachtet und den Willen zur Selbsthilfe [...] lähmt“. ${ }^{141}$ Dadurch stärke der Entwurf „die Allmacht des Staates und erweitert die staatlich dirigierten Sozialhilfen in einem Umfang, der uns die Grundlagen unseres demokratischen Gemeinwesens anzutasten scheint". Die Worte Gerstenmaiers waren also auch im DLT vernommen worden. Dessen Vertreter beriefen sich vor allem auf die besondere ländliche Situation, wo die familiäre und nachbarschaftliche Hilfe noch lebendig sei und wegen des niedrigeren Lebensstandards Leistungen, die im großstädtischen Umfeld vielleicht notwendig seien, oft unan-

sterien und Kommunalvertretungen der Länder vgl. Schreiben des Städteverbands Baden-Württemberg an die DST-Hauptgeschäftsstelle vom 7. 8.1958, des nordrhein-westfälischen Sozialministeriums an den dortigen Städtetag und den dortigen Landkreistag vom 8. 8.1958 sowie die Antwort des Landkreistages Nordrhein-Westfalen vom 15.8. 1958, LAB, B Rep. 142-9, 1283.

137 Teilweise ähnlich die Stellungnahme der beim Landschaftsverband Westfalen-Lippe angesiedelten AG der LFV vom 17.12.1958, BAK, B 106/20644; Schreiben des Direktors des Landschaftsverbands Köchling an den Städtetag Nordrhein-Westfalen, 31.12.1958, LAB, B Rep. 142-9, 1284.

138 Ziebill an Abteilung V des BMI, 1.12.1958, BAK, B 106/9686.

139 Stellungnahme des DST vom 27.11.1958, ebenda. Diese Stellungnahme war wieder vom Arbeitskreis bzw. dem Sozialausschuß vorbereitet worden; vgl. die Niederschriften über die Sitzungen des Arbeitskreises „Fürsorgerecht“ am 22./23.9.1958 (mit Arbeitsunterlage) sowie des DST-Sozialausschusses (Auszug) am 3./4.10.1958, LAB, B Rep. 142-9, 1283. Vgl. auch Oel, Neuordnung des Fürsorgerechts.

140 Schreiben des Geschäftsführers des Landesverbandes Schleswig-Holstein des DST an Oel, 25.11.1958, LAB, B Rep. 142-9, 1284.

141 Vgl. auch zum Folgenden die Stellungnahme des DLT vom 17.11.1958, BAK, B 106/9686. 
gemessen seien. Der Gesetzgeber wolle „es offenbar unternehmen, das ganze dynamische und bunte Leben in gesetzlich normierten Tatbeständen einzufangen“. Dieser "Gesetzesperfektionismus" mit seinen nivellierenden Leistungsvorgaben werde nicht nur den unterschiedlichen finanziellen Möglichkeiten der Träger nicht gerecht, sondern führe zu den „größten sozialen Ungerechtigkeiten“. Wenn somit der DLT eigentlich den Gesetzentwurf prinzipiell ablehnte und lieber einen Großteil der geplanten Vorschriften in weniger verbindlichen „Bundesgrundsätzen“ oder Verwaltungsvorschriften untergebracht hätte, hatte man sich auf Betreiben der Hauptgeschäftsstelle im Präsidium doch dazu durchgerungen, auch zu den Einzelregelungen Stellung zu nehmen, um sich durch bloße Obstruktion nicht ins Abseits zu manövrieren. ${ }^{142}$ Der DLT forderte daher eine deutliche Einschränkung der Leistungsansprüche und -inhalte, niedrigere Einkommensgrenzen, die Beibehaltung bestehender Unterhalts- und Ersatzpflichten und flexiblere Richtsätze. Diese angesichts der bereits evidenten tiefgreifenden Wandlungen der ländlichen Sozialstruktur etwas anachronistisch anmutenden Argumente ${ }^{143}$ machten nicht nur auch die im DGT organisierten kreisangehörigen Gemeinden und die bayerischen kommunalen Spitzenverbände geltend; auch die Kommunalreferate des Bundesinnenministeriums griffen sie z.T. wörtlich auf und stärkten so die Phalanx der Gegner einer weiter ausgreifenden Fürsorgereform. ${ }^{144}$

Die Situation entbehrte damit nicht einer gewissen Pikanterie: Während der sozialdemokratisch geprägte DST die Reformlinie des CDU-geführten Innenministeriums weitgehend stützte, ja diese inhaltlich sogar deutlich beeinflußt hatte, kam die heftigste Opposition von seiten des christdemokratisch dominierten Landkreistages. Die häufig von Kommunalfunktionären geäußerte Klage, daß kommunale Interessen von den Bundespolitikern im Zweifelsfalle der Parteiräson geopfert würden ${ }^{145}$, hatte also durchaus ihre umgekehrte Entsprechung bei den Kommunalfunktionären.

Daß die vehemente Kritik der Landkreise bei Duntze und Gottschick Früchte trug, lag allerdings in erster Linie daran, daß auch das Bundesfinanzministerium grundlegende Einwände erhob. Laut Geschäftsordnung der Bundesregierung war

142 Vgl. auch Johann Bangert, Landkreise; Heisig, Armenpolitik, 1995, S. $158 f$.

143 Vgl. Ritter, Über Deutschland, S.77ff. Auch Bangert sah in dieser Argumentation einen Widerspruch zu der in anderen Bereichen geforderten Gleichberechtigung von Stadt und Land und nach Angleichung des Lebensstandards; die Berufung auf die besonderen Verhältnisse wirke "gegenüber unserer sonstigen Tendenz antiquiert“, Bangert im Mai 1961, zit. bei Heisig, Armenpolitik, 1995, S. 177.

144 Vgl. Bayerischer Städteverband und Landkreisverband Bayern an das Bayerische Staatsministerium des Innern, 14.10.1958; Stellungnahme des DGT vom 1.12.1958; Referate I C 5 und I C 7 an Referat V 4, 22.12.1958, BAK, B 106/9686; Heisig, Armenpolitik, 1995, S. 158ff. Die Stellungnahme des DSB, die Heisig 1990 offenbar zur Verfügung stand, konnte leider von der Verfasserin archivalisch nicht mehr nachgewiesen werden. Laut Heisig, Armenpolitik, 1995, S.158, lag sie in ihrer Argumentation zwischen der des DST und des DLT. Angesichts der Stellungnahme des DSB-Beigeordneten Possehl vor dem Bundestagsausschuß für Kommunalpolitik und öffentliche Fürsorge im Juni 1960 ist davon auszugehen, daß der DSB als Vertretung der kreisangehörigen Städte insgesamt der DLT-Linie näherstand.

145 Vgl. Bertram, Staatspolitik, S. $148 f f$. 
schon vor der Kabinettsreife eines Entwurfs das Votum dieses Ministeriums einzuholen, sofern das Gesetzesvorhaben Bund, Länder oder Gemeinden belastete. ${ }^{146} \mathrm{Da}$ der Finanzminister in solchen Fällen im Kabinett de facto ein absolutes Vetorecht hatte, war schon deshalb auf solche Einwände Rücksicht zu nehmen. ${ }^{147}$ Eigentlich hatte der Bund bei der durch die Kommunen und gegebenenfalls durch die Länder zu finanzierenden Fürsorge, zumal nach der Pauschalierung der Kriegsfolgenhilfe, nur sehr beschränkte eigene Mehrausgaben (Kriegsopferfürsorge, nicht pauschalierte Kriegsfolgenhilfe für Zugewanderte aus der DDR, Bundesanteile der Tuberkulosefürsorge) zu gewärtigen. Doch im Finanzministerium fürchtete man, daß die Länder aufgrund der zu erwartenden neuen Belastungen vom Bund erhöhte Pauschalleistungen und zusätzliche Gelder im Rahmen des vertikalen Finanzausgleichs nach dem neu gefaßten Art.106 GG fordern würden. ${ }^{148}$ Tatsächlich hatten DLT-Vertreter entsprechende Wünsche im Finanzministerium bereits angekündigt. ${ }^{149}$ Elsholz erklärte, daß sein Minister diese Wünsche ablehnen werde. Um die Mehrkosten der neuen Sozialhilfe zu senken, forderte er daher eine deutliche Lockerung der Leistungsverpflichtungen des auch in seinen Augen „zu perfektionistischen“ Entwurfs ${ }^{150}$ - ein abermaliger Beleg für die Beobachtung, daß das Bundesfinanzministerium zwar selbst von Verbandsdruck weitgehend unabhängig gewesen sei, sich diesen aber sehr wohl im Interesse eigener Ziele taktisch zunutze machte. ${ }^{151}$

Eine weitere Institution, von der ebenfalls wesentlicher Einfluß auf die weitere Reformarbeit der Sozialabteilung vermutet werden konnte, blieb in ihrer Stellungnahme hingegen bemerkenswert diffus: Der DV übersandte kein geschlossen konzipiertes Gutachten seines Vorstands, sondern ein umfangreiches Konvolut von „Äußerungen aus den Fachgremien und der Geschäftsstelle“ des DV, in dem zu jedem Paragraphen Änderungsvorschläge oder auch nur Diskussionspunkte aufgeführt wurden. ${ }^{152}$ Infolge der im DV und in dessen Vorstand versammelten heterogenen Interessen wäre eine solche Stellungnahme auch kaum möglich gewesen: Allein die Beratungen des unter Collmers Vorsitz wiederbelebten Fachausschusses I hatten gezeigt, daß etwa bei den Fragen der Stellung der freien Wohlfahrtspflege oder der Rückerstattungspflicht keine einheitliche Position zu finden

146 Vgl. $\ 16$ der Geschäftsordnung der Bundesregierung vom 11. 5. 1951, GMBl. S. 137.

147 Vgl. allgemein Hockerts, Entscheidungen, S. $119 \mathrm{f}$.

148 Vgl. Vermerk Referat V 4 vom 11.12.1958, BAK, B 106/9686.

149 Vgl. Heisig, Armenpolitik, 1995, S. 161.

150 Die übrigen betroffenen Bundesministerien für Arbeit, für Wirtschaft und für Vertriebene wünschten dagegen jeweils nur ressortspezifische Modifikationen (der Fall des Familienministeriums lag anders); vgl. Vermerk Referat V 4 vom 23.10.1958 über eine Besprechung mit Vertretern des BMA, BAK, B 106/9686, sowie die Stellungnahmen des Bundesministers für Vertriebene, Flüchtlinge und Kriegsgeschädigte vom 28.10.1958, BAK, B 106/20644, und des Bundesministers für Wirtschaft vom 4.12.1958, BAK, B 106/9686.

151 Vgl. Hockerts, Entscheidungen, S.120; Heisig, Armenpolitik, 1995, S.158ff., allerdings deutet die Konzessionsbereitschaft des BMI vor allem als Ergebnis eines „erfolgreichen Gegenstoßes des kommunalen Traditionalismus“.

152 Vgl. Äußerungen aus den Fachgremien und der Geschäftsstelle des DV, mit Schreiben von Muthesius vom 5.11.1958 an das BMI übersandt, BAK, B 106/9686. 
war. ${ }^{153}$ Ebensowenig waren die Forderungen einzelner Fachausschüsse nach höheren Leistungsansprüchen mit denen vieler Kommunalvertreter vereinbar. Damit erwies sich, daß „der“ DV - anders als in der unmittelbaren Nachkriegszeit unter Führung Polligkeits - nicht als wirklich eigenständiger Akteur im Gesamtprozeß der Fürsorgereform zu betrachten war; vielmehr agierten darin, nicht selten in ganz unterschiedliche Richtung, einzelne seiner Mitglieder und Gremien, denen es aber zum Teil mit Erfolg gelang, die Fiktion eines durch die fachliche Autorität „des" DV gedeckten Vorgehens zur eigenen Legitimation aufrechtzuerhalten.

Wie unterschiedlich die unter dem Dach des DV versammelten Anliegen waren, offenbarten auch die Stellungnahmen der Spitzenverbände der freien Wohlfahrtspflege und Fachorganisationen, die eher ein Zuwenig als ein Zuviel an Leistungen konstatierten und meist weitere Verbesserungen für ihre jeweilige Klientel forderten. Einzig der DPW näherte sich bisweilen bemerkenswert den kommunalen Einwänden an, wenn er eine stärkere Betonung der Selbsthilfe- und der Unterhaltspflicht wünschte. ${ }^{154}$ Die AWO hingegen forderte, daß jeder Staatsbürger einen Rechtsanspruch auf alle Hilfen in besonderen Lebenslagen haben und nur entsprechend seiner finanziellen Mittel gegebenenfalls dazu einen Kostenbeitrag leisten solle. ${ }^{155}$ Die mittlerweile fusionierten evangelischen Wohlfahrtsverbände bewerteten den Entwurf weitgehend positiv und wünschten nur noch punktuelle Leistungsverbesserungen. ${ }^{156}$ Wesentlicher Kritikpunkt war hier, daß die Stellung der freien Wohlfahrtspflege im Gesetz nicht genügend geschützt sei. Der Caritasverband behandelte sogar fast ausschließlich diese Frage und forderte eine klare Privilegierung der freien Wohlfahrtsverbände in einem Fürsorgegesetz, das die persönlichen Hilfen und damit ihre klassische Domäne künftig in den Mittelpunkt stellen wollte. ${ }^{157}$

Im Januar 1959 legte Gottschick eine gründlich überarbeitete, deutlich gestraffte und in vielen Bereichen für den Hilfeempfänger weniger günstige Version des Entwurfs vor, die nun auch auf positive Resonanz der kommunalpolitischen Spezialisten der Länder stieß. ${ }^{158}$ Mittlerweile distanzierte sich Duntze öffentlich von dem ersten Referentenentwurf, wenn er ihn nur mehr als "Diskussionsgrundlage“ bezeichnete, die „in keiner Weise gleichbedeutend mit einer Kabinettsvorlage“ sei. 159 Die breite Anfrage von Stellungnahmen hatte also - anders als die Einbeziehung vieler Experten und Fachgremien zuvor - nicht zu einer Absicherung des Entwurfs des Innenministeriums geführt, sondern zu dessen gründlicher Revi-

153 Vgl. die Berichte über die Sitzungen des DV-Fachausschusses I am 8.10.1958, ADW, HGSt 2486, und 24.10.1958, ADW, HGSt, SP-S XXIIIc I/1.

154 Vgl. die DPW-Stellungnahme vom 22.12.1958, BAK, B 106/9686.

155 Vgl. die AWO-Stellungnahme vom 14.10.1958; ähnlich die vor allem am SPD-Sozialplan orientierte, allgemein gehaltene Stellungnahme des DGB vom 4.11.1958, ebenda.

156 Vgl. die Stellungnahme von Innerer Mission und Hilfswerk der EKD vom 14.11.1958, ebenda.

157 Vgl. die DCV-Stellungnahme vom 30. 8.1958, ebenda.

158 Vgl. den BSHG-Entwurf vom Januar 1959, BAK, B 106/20646; Vermerk Referat V 4 vom 15.1.1959 über eine Beratung des Arbeitskreises III „Kommunale Angelegenheiten“ der Arbeitsgemeinschaft der Innenministerien der Bundesländer, BAK, B 106/9686.

159 KommBl 11 (1959), S. 187. 
sion. Hinweise darauf, daß dies von Anfang an von Duntze und/oder Gottschick beabsichtigt war, daß man also in geradezu machiavellistischer Manier zunächst Maximalregelungen formuliert und diese dann mit der Rückendeckung der kritischen Stellungnahmen in längst beabsichtigter Weise verändert habe, gibt es nicht. Die ursprüngliche Zeitplanung spricht eher dagegen. $\mathrm{Zu}$ vermuten ist vielmehr, daß die Sozialabteilung, und hier vor allem Scheffler, den Reformwillen, gerade auch auf der Ebene der Ländervertreter und innerhalb des DST überschätzt hatte. Am 23. März 1959 jedenfalls legte Duntze seinem Minister den nun auch mit einer ausführlichen Begründung versehenen offiziellen zweiten Referentenentwurf vor. ${ }^{160}$

160 Vgl. Ministervorlage vom 23.3.1959, BAK, B 106/20647, und „Entwurf eines Gesetzes über die Gewährung von Sozialhilfe" vom März 1959, im folgenden: BSHG-Entwurf 3/1959, BAK, B 106/20646. 